\title{
Bulk gauge and matter fields in nested warping: I. The formalism
}

\author{
Mathew Thomas Arun ${ }^{1}$ and Debajyoti Choudhury \\ Department of Physics and Astrophysics, University of Delhi, \\ Delhi 11000\%, India \\ E-mail: thomas.mathewarun@gmail.com, debajyoti.choudhury@gmail.com
}

\begin{abstract}
The lack of evidence for a TeV-mass graviton has been construed as constricting the Randall-Sundrum model. However, a doubly-warped generalization naturally avoids such restrictions. We develop, here, the formalism for extension of the Standard Model gauge bosons and fermions into such a six-dimensional bulk. Apart from ameliorating the usual problems such as flavour-changing neutral currents, this model admits two very distinct phases, with their own unique phenomenologies.
\end{abstract}

Keywords: Field Theories in Higher Dimensions, Gauge Symmetry, Spontaneous Symmetry Breaking, Intersecting branes models

ArXiv EPrint: 1501.06118

\footnotetext{
${ }^{1}$ Corresponding author.
} 


\section{Contents}

1 Introduction 1

2 Brief review: nested warping in six dimensions 3

3 The fermions $\quad 6$

3.1 Flat six-dimensions 6

$\begin{array}{lll}3.2 & \text { Fermions in the warped space } & 7\end{array}$

$\begin{array}{lll}3.3 & \text { KK masses for the fermions } & 10\end{array}$

$\begin{array}{lll}3.3 .1 & \text { Small } k \text { and large } c & 10\end{array}$

$\begin{array}{lll}3.3 .2 & \text { Large } k \text { and small } c & 11\end{array}$

4 The gauge bosons $\quad 11$

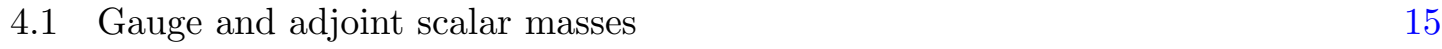

$\begin{array}{lll}\text { 4.1.1 Boundary conditions in the } x_{4} \text {-direction } & 16\end{array}$

$\begin{array}{ll}\text { 4.1.2 Boundary conditions in the } x_{5} \text {-direction } & 16\end{array}$

$\begin{array}{lll}\text { 4.1.3 Small } k \text { and large } c & 17\end{array}$

$\begin{array}{lll}\text { 4.1.4 Large } k \text { and small } c & 19\end{array}$

4.1.5 Bulk masses 20

$\begin{array}{lll}4.2 \text { Ghosts } 20 & 20\end{array}$

5 Higgs $\quad 21$

5.13 -brane localization 22

5.2 4-brane localization 23

6 Interactions $\quad \mathbf{2 5}$

6.1 Gauge-fermion 25

6.2 Triple gauge boson couplings 27

$\begin{array}{ll}6.3 \text { Quartic gauge interaction } & 29\end{array}$

$\begin{array}{lll}6.4 & \text { Ghost vertices } & 29\end{array}$

$\begin{array}{lll}7 & \text { Summary } & 30\end{array}$

\section{Introduction}

The spectacularly successful Standard Model (SM) has received a recent fillip with the discovery of the long-sought for Higgs boson [1-3]. Yet, certain questions remain unanswered. These pertain to the existence of Dark Matter, the origin of the baryon asymmetry in the Universe, the existence of multiple generations of fermions, the hierarchy in fermion masses and mixing, and, last but not the least, the stability of the Higgs sector under quantum 
corrections. Over the years, several attempts have been made to answer these questions, albeit only with partial success. One such stream of thought envisages a world in more than three space dimensions as a possible panacea to some of the ills of the SM, and, in this paper, we concentrate on this possibility.

While such theories were first proposed nearly a century ago [4-6] in the quest to unify electromagnetism and gravity, the early efforts were quickly shown to lead to a dead end and were abandoned. The situation changed with the introduction of String Theory as a quantum theory of gravity as well as a possible ultraviolet completion of the SM. With the theory defined, of necessity, in at least ten dimensions, compactification of the extra dimensions is paramount before it can be deemed a description of the observed world. With the compactification scale being close to the Planck scale in most early constructions, the new dimensions, understandably played virtually no role in low-energy physics. However, warped compactification $[7,8]$, wherein the SM fields were confined to the usual $(3+1)$ dimensions with only gravity being allowed to propagate in the (five-dimensional) bulk, led not only to a "resolution" of the hierarchy problem, but also to interesting consequences at colliders owing to distinct $\mathcal{O}(\mathrm{TeV})$ resonances in the form of Kaluza-Klein (KK) excitations of the graviton.

The last feature moved both the ATLAS [9] and CMS [10] experiments to investigate the existence of such Randall-Sundrum (RS) graviton resonances, especially through the dilepton and diphoton decay channels. In particular, the ATLAS experiment ruled out graviton masses below $1.03(2.23) \mathrm{TeV}$ at 95\% C.L. with the lower bound being dependent on the ratio of the five-dimensional curvature and the fundamental mass scale. This ratio is constrained, on the upper side, by the applicability of a semiclassical treatment (only recourse available in the absence of a full quantum theory of gravity) and, on the lower, by the undesirability of fine-tuning. For reasonable values of this ratio, the mass of the first graviton excitation should, preferably, be a few times that of the Higgs boson; and certainly no higher than a few TeVs. Thus, the continued absence of any such resonance at the $\mathrm{TeV}$ scale begins to call into question the validity of this scenario as a cure for the Higgs mass stabilization problem. However, it should be realized that the RS model is only the simplest of possible warped world scenarios. In particular, there is no reason that there should be only one such extra dimension [11-31]. For one, in a scenario with double (or more) warping [31], it was shown recently that the aforementioned ATLAS bounds are naturally evaded [32]. This motivates us to study the features of six-dimensional theories.

Although the mechanism for the formation of branes and the localization of fermions thereon is well understood, it is interesting to consider allowing them to propagate in the full six dimensions. The corresponding flat space theories have several interesting consequences. For example, the analogue of Witten anomaly cancellation leads to a prediction of the number of chiral generations [33], while suppressing the proton decay rate to below the current constraints [34]. Furthermore, some of these constructions [35] naturally lead to a small cosmological constant. The rich collider phenomenology [36-39], apart from the existence of a viable cold dark matter candidate [40-42] renders these scenarios phenomenologically attractive. On the other hand, with the KK excitations for each species now expanding to a "tower of towers", the quantum corrections to the SM amplitudes 
- most importantly to the electroweak precision variables - are potentially large, calling into question the consistency with low-energy phenomenology. However, as ref. [43] demonstrated for a five-dimensional theory, it is possible to suppress the coupling between the zero modes of the SM fields and the KK towers. This is of particular importance in the context of the aforementioned quantum corrections. Thus, it is of interest to investigate whether considering a warped space would allow us to preserve some of the advantages of going into six dimensions while simultaneously protecting us from the pitfalls. This paper is the first step towards this goal, and we set up the entire formulation here and comment on some of the consequences. While ref. [45] did consider bulk SM fields in such a geometry, the analysis therein had taken recourse to an approximation of the metric, thereby leading to a significant simplification of the equations of motion. However, as was demonstrated in ref. [32], the said approximation, apart from being untenable close to the brane we live on, led to a drastic change in the form of the graviton wavefunctions (and, hence, their couplings). As we shall show, much the same happens for bulk gauge bosons as well, leading to very interesting phenomenological consequences. The detailed phenomenology would be presented in subsequent papers. ${ }^{1}$

The rest of the article is constructed as follows. To begin with, we present a very brief review of the doubly warped space. Sections $3 \& 4$ discuss, respectively, the bulk fermions and gauge bosons in this theory, without taking into consideration the spontaneous breaking of the gauge symmetry which, in turn, is discussed in section 5 . The interactions are delineated in section 6 and the Feynman rules listed. Finally, we conclude in section 7.

\section{Brief review: nested warping in six dimensions}

We consider a compactified six-dimensional space-time with successive warpings and $Z_{2}$ orbifolding in each of the two extra dimensions, viz. $M^{1,5} \rightarrow\left[M^{1,3} \times S^{1} / Z_{2}\right] \times S^{1} / Z_{2}$. Dual requirements of nested warping along with a manifestly exhibited four-dimensional $\left(x^{\mu}\right)$ Lorentz symmetry restricts the line element to the form [31]

$$
d s^{2}=b^{2}\left(x_{5}\right)\left[a^{2}\left(x_{4}\right) \eta_{\mu \nu} d x^{\mu} d x^{\nu}+R_{y}^{2} d x_{4}^{2}\right]+r_{z}^{2} d x_{5}^{2},
$$

where the compact directions are represented by the dimensionless coordinates $x_{4,5} \in[0, \pi]$ with $R_{y}$ and $r_{z}$ being the corresponding moduli. It is interesting to examine the rationale for the two orbifoldings. A nontrivial $a\left(x_{4}\right)$, when accompanied by compactification, demands (as in the RS case), the orbifolding in the $x_{4}$-direction. Furthermore, it necessitates the presence of localized energy densities at the orbifold fixed points, and in the present case, these appear in the form of tensions associated with the two end-of-the-world 4-branes at $x_{4}=0, \pi$. Similarly, even without any orbifolding in the $x_{5}$-direction, a nontrivial $b\left(x_{5}\right)$ for a compactified $x_{5}$ automatically requires that a 4 -brane should exist at $x_{5}=\pi$, whereas none needs to exist at $x_{5}=0$. The situation changes though if one wishes to introduce such a brane. While the latter could exist even in the absence of such an orbifolding, it would

\footnotetext{
${ }^{1}$ It should be pointed out that six dimensional warped models with spherical compactifcations [46, 47], do try to explain the number of fermion families [48]. However, with these models having only a single warping, the aforementioned constraints on the RS scenario continue to hold, albeit with some modifications.
} 
be free to traverse in the $x_{5}$-direction in the absence of a constraining potential. Thus, if such a brane is to be introduced, it is easiest to do so if the second $S^{1}$ is orbifolded too.

The total bulk-brane action for the six dimensional space time is, then, given by

$$
\begin{aligned}
\mathcal{S}= & \mathcal{S}_{6}+\mathcal{S}_{5} \\
\mathcal{S}_{6}= & \int d^{4} x d x_{4} d x_{5} \sqrt{-g_{6}}\left(M_{6}^{4} R_{6}-\Lambda\right) \\
\mathcal{S}_{5}= & \int d^{4} x d x_{4} d x_{5} \sqrt{-g_{5}}\left[V_{1}\left(x_{5}\right) \delta\left(x_{4}\right)+V_{2}\left(x_{5}\right) \delta\left(x_{4}-\pi\right)\right] \\
& +\int d^{4} x d x_{4} d x_{5} \sqrt{-\tilde{g}_{5}}\left[V_{3}\left(x_{4}\right) \delta\left(x_{5}\right)+V_{4}\left(x_{4}\right) \delta\left(x_{5}-\pi\right)\right],
\end{aligned}
$$

where $\Lambda$ is the (six dimensional) bulk cosmological constant and $M_{6}$ is the fundamental scale (quantum gravity scale) in six dimensions. The five-dimensional metrics in $\mathcal{S}_{5}$ are those induced on the appropriate 4-branes which lend a rectangular box shape to the space.

If the bulk cosmological constant $\Lambda$ is negative, the solutions for the 6 -dimensional Einstein field equations are given by [31]

$$
\begin{aligned}
a\left(x_{4}\right) & =e^{-c\left|x_{4}\right|} & c & =\frac{R_{y} k}{r_{z} \cosh k \pi} \equiv \frac{\alpha k}{\cosh (k \pi)} \\
b\left(x_{5}\right) & =\frac{\cosh \left(k x_{5}\right)}{\cosh (k \pi)} & k & =r_{z} \sqrt{\frac{-\Lambda}{10 M_{6}^{4}}} \equiv \epsilon r_{z} M_{6},
\end{aligned}
$$

where we have introduced the dimensionless constants $\alpha$ and $\epsilon$ for future reference. Clearly, the validity of the semiclassical treatment (to the extent of neglecting quantum corrections to the bulk gravity action) requires the bulk curvature to be significantly smaller than the fundamental scale $M_{6}$ and it has been argued in the literature to imply that $\epsilon \lesssim 0.1$. Similarly, the ratio of the two moduli should not be too large so as to not reintroduce a large hierarchy.

As in the RS scenario, the brane tensions in eq. (2.2) are specified by the junction conditions. The smoothness of the warp factor at $x_{5}=0$ obviates the necessity for a $V_{3}\left(x_{4}\right)$, while the fixed point at $x_{5}=\pi$ requires a negative tension, viz.

$$
V_{3}\left(x_{4}\right)=0, \quad V_{4}\left(x_{4}\right)=\frac{-8 M_{6}^{4} k}{r_{z}} \tanh (k \pi) .
$$

In contrast, the two 4 -branes sitting at $x_{4}=0$ and $x_{4}=\pi$ require equal and opposite energy densities, just as in the RS case. However, the $x_{5}$-warping dictates that, rather than being constants, these energy densities must be $x_{5}$-dependent, viz.

$$
V_{1}\left(x_{5}\right)=-V_{2}\left(x_{5}\right)=8 M_{6}^{2} \sqrt{\frac{-\Lambda}{10}} \operatorname{sech}\left(k x_{5}\right) .
$$

Such tensions could originate from different kinds of physics [31]. The most simple would be a scalar field with a non trivial potential, wherein the solution of eq. (2.3), along with the expressions for $V_{1,2}\left(x_{5}\right)$, is nothing but a self consistent solution for the gravity-scalar system. While a wide variety of potentials can have such a kink-like solution [31], it is 


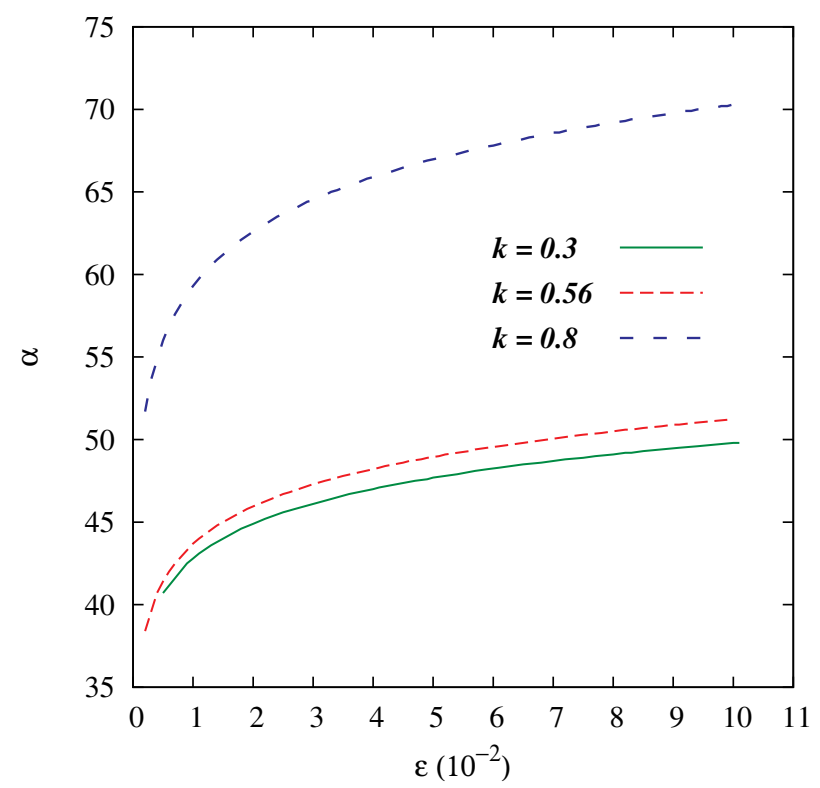

Figure 1. Contour plots in the $(\epsilon, \alpha)$ plane for fixed values of $k$ with $R_{y}$ set to satisfy the hierarchy eq. (2.7).

intriguing to note that even a simple quartic form can do the job. (In section 5, we shall encounter a variant of this mechanism.) And while other scenarios, such as a Born-Infeld action, can also lead to a similar effective potential, we shall not explore those here.

The (derived) 4-dimensional Planck scale can be related to the fundamental scale $M_{6}$ through

$$
M_{\text {Planck }}^{2} \sim \frac{M_{6}^{4} r_{z} R_{y}}{2 c k}\left(1-e^{-2 c \pi}\right)\left[\frac{\tanh k \pi}{\cosh ^{2} k \pi}+\frac{\tanh ^{3} k \pi}{3}\right] .
$$

The point in this 2-dimensional $\left(x_{4}-x_{5}\right)$ plane associated with the lowest energy scale is given by $x_{4}=\pi, x_{5}=0$. Assuming us to be located at this juncture immediately gives the required hierarchy factor (i.e., the mass rescaling due to warping) to be

$$
w=\frac{e^{-c \pi}}{\cosh k \pi} .
$$

For the large $w$ that we need, this equation, along with the relation between $c$ and $k$ (eq. (2.3)) demands that, unless there is a very large hierarchy between the moduli, the warping is substantial in only one of the two directions, and rather sub-dominant in the other. In other words, we can have either $(i)$ a large $(\sim 10)$ value for $k$ accompanied by an infinitesimally small $c$ or $(i i)$ a large $(\sim 10)$ value for $c$ with a moderately small $(\lesssim 1.0) k$. The relationship between the parameters of the theory are displayed in figure 1 .

In summary, we are dealing with a brane world which is doubly warped, with the warping being large along one direction and small in the other. The very structure of the theory typically requires a small hierarchy between the two moduli, both of which remain comparable to the fundamental length scale in the theory. It should be realized that the two branches, namely $(i)$ a large $k$ and a small $c$, or $(i i)$ a large $c$ and a small $k$, are fundamentally different. 


\section{The fermions}

As is well-known, fermions, of necessity, are defined as representations of the Poincare algebra, as applicable to the tangent space. Hence, we begin by briefly reviewing the construction in flat space before embarking on the more germane issue of the warped space.

\subsection{Flat six-dimensions}

In the current case, the spin- $1 / 2$ representation is defined by six $8 \times 8$ matrices $\Gamma^{a}$, satisfying a Clifford algebra

$$
\left\{\Gamma_{a}, \Gamma_{b}\right\}=2 \eta_{a b}
$$

where $\eta_{a b}=\operatorname{diag}(-1,+1,+1,+1,+1,+1)$. We choose to work with a particular representation of the algebra defined by

$$
\begin{array}{ll}
\Gamma_{\hat{\mu}}=\gamma_{\hat{\mu}} \otimes \sigma_{3} & \Gamma_{4}=1 \otimes \sigma_{1} \\
\Gamma_{5}=1 \otimes \sigma_{2} & \Gamma_{7}=\gamma_{5} \otimes \sigma_{3},
\end{array}
$$

where $\gamma_{\hat{\mu}}$ (with $\hat{\mu}$ denoting the subspace of the flat space) are the four-dimensional Dirac matrices (in the Weyl representation) and $\gamma_{5}\left(\Gamma_{7}\right)$ is the parity operator in four (six) dimensions. ${ }^{2}$ The direct product is defined in a trivial sense and, for example,

$$
\gamma_{\hat{\mu}} \otimes \sigma_{3}=\left(\begin{array}{rl}
\gamma_{\hat{\mu}} & 0 \\
0 & -\gamma_{\hat{\mu}}
\end{array}\right) .
$$

It is straightforward to construct the Dirac spinor $\Psi\left(x_{\hat{\mu}}, x_{4}, x_{5}\right)$ satisfying the flat space Dirac equation

$$
\left[\Gamma_{\hat{\mu}} \partial^{\hat{\mu}}+\Gamma_{4} \partial_{4}+\Gamma_{5} \partial_{5}-m\right] \Psi\left(x_{\hat{\mu}}, x_{4}, x_{5}\right)=0 .
$$

The representation of the Lorentz generators given by $\Sigma_{a b}=\frac{i}{2}\left[\Gamma_{a}, \Gamma_{b}\right]$, viz.

$$
\begin{array}{rlrl}
\Sigma_{\hat{\mu} \hat{\nu}} & =\frac{i}{2}\left[\Gamma_{\hat{\mu}}, \Gamma_{\hat{\nu}}\right]=S_{\hat{\mu} \hat{\nu}} \otimes \sigma_{0} & \Sigma_{\hat{\mu} 4}=\frac{i}{2}\left[\Gamma_{\hat{\mu}}, \Gamma_{4}\right]=-\gamma_{\hat{\mu}} \otimes \sigma_{2} \\
\Sigma_{\hat{\mu} 5}=\frac{i}{2}\left[\Gamma_{\hat{\mu}}, \Gamma_{5}\right]=\gamma_{\hat{\mu}} \otimes \sigma_{1} & \Sigma_{45}=\frac{i}{2}\left[\Gamma_{4}, \Gamma_{5}\right]=1 \otimes \sigma_{3}
\end{array}
$$

is, of course, reducible, as is the case for all even dimensions. In other words, this space admits chiral representations $\Psi_{ \pm}$and thus, we may directly export the SM quantum number assignments. This is quite unlike the five-dimensional case. Of course, on compactification, each such six-dimensional chiral representation would, in general, yield low-lying states carrying either value for the four-dimensional chirality unless boundary conditions (such as those pertaining to the orbifold fixed points) prevent this. Before we consider such details, we need to set up the Dirac equation in the warped six-dimensional space which we do next.

\footnotetext{
${ }^{2}$ Note that this implies that $\Gamma_{0}$ is antihermitian, while the other $\Gamma_{M}$ are hermitian.
} 


\subsection{Fermions in the warped space}

To define these, we need to consider the sechsbeins (namely, the transformations to the tangent space) $e_{M}^{a}$ which satisfy the conditions

$$
e_{M}^{a} e_{N}^{b} g^{M N}=\eta_{a b}, \quad e_{M}^{a} e_{N}^{b} \eta^{a b}=g_{M N},
$$

leading to

$$
e_{\mu}^{a}=a\left(x_{4}\right) b\left(x_{5}\right) \delta_{\mu}^{a}, \quad e_{4}^{a}=R_{y} b\left(x_{5}\right) \delta_{4}^{a}, \quad e_{5}^{a}=r_{z} \delta_{4}^{a} .
$$

Denoting the inverse sechsbeins by $E_{a}^{M}$, we define the spin connections $\omega_{b c M}$ through the covariant derivatives of $E_{a}^{M}$, viz.,

$$
\omega_{b c M} \equiv E_{b}^{N}\left(g_{P N} E_{c}^{P}\right)_{; M}=g_{R N} E_{b}^{N}\left(\partial_{M} E_{c}^{R}+\Gamma_{M T}^{R} E_{c}^{T}\right) .
$$

For the metric of eq. (2.1), the only nontrivial components of the spin connections are given by

$$
\omega_{b c 4}=\frac{R_{y}}{r_{z}} \dot{b} \delta_{[b}^{5} \delta_{c]}^{4}, \quad \omega_{b c \mu}=\eta_{\mu \nu}\left(\frac{a^{\prime}}{R_{y}} \delta_{[b}^{\nu} \delta_{c]}^{4}+\frac{a \dot{b}}{r_{z}} \delta_{[b}^{\nu} \delta_{c]}^{5}\right),
$$

where primes (dots) denote derivatives with respect to $x_{4}\left(x_{5}\right)$. The Dirac Lagrangian in the warped geometry is, then, given by

$$
\mathcal{L}_{\text {Dirac }}=i \bar{\Psi}_{+} \Gamma^{a} E_{a}^{M}\left(\partial_{M}+w_{M}^{b c}\left[\Gamma_{b}, \Gamma_{c}\right]\right) \Psi_{+}
$$

for the positive chirality field $\Psi_{+}$and, analogously, for $\Psi_{-}$as well. The corresponding equation of motion is

$$
\Gamma^{a} E_{a}^{M} D_{M} \Psi_{+}=\left[\left(\frac{\Gamma^{\mu}}{a b} \partial_{\mu}+\frac{\Gamma^{4}}{R_{y} b} \partial_{4}+\frac{\Gamma^{5}}{r_{z}} \partial_{5}\right)+\frac{1}{2}\left(4 \Gamma^{4} \frac{a^{\prime}}{a b R_{y}}+5 \Gamma^{5} \frac{\dot{b}}{b r_{z}}\right)\right] \Psi_{+}=0 .
$$

Anticipating Kaluza-Klein reduction, we write the positive chirality Weyl spinor as

$$
\Psi_{+}=\frac{1}{\sqrt{R_{y} r_{z}}} \sum_{n, p}\left[\mathcal{F}_{l}^{n, p}\left(x_{4}, x_{5}\right) \psi_{l}^{n, p}\left(x_{\mu}\right) \otimes \mathcal{S}_{\mathrm{up}}+\mathcal{F}_{r}^{n, p}\left(x_{4}, x_{5}\right) \psi_{r}^{n, p}\left(x_{\mu}\right) \otimes \mathcal{S}_{\mathrm{dn}}\right],
$$

with

$$
\mathcal{S}_{\text {up }} \equiv\left(\begin{array}{ll}
1 & 0
\end{array}\right)^{T}, \quad \mathcal{S}_{\text {dn }} \equiv\left(\begin{array}{ll}
0 & 1
\end{array}\right)^{T} .
$$

Here, $\mathcal{F}_{r}^{n, p}\left(x_{4}, x_{5}\right)$ encapsulate the wavefunction dependences on the extra dimensions, with the subscripts $(l, r)$ referring to the (four-dimensional) chirality of the putative fourdimensional fields $\psi_{l, r}^{n, p}$ whereas the factor $\sqrt{R_{y} r_{z}}$ (ensuring the correct mass dimension) would have arisen if the compactified directions were flat instead. The Dirac equation then reduces to

$$
\begin{aligned}
0= & {\left[\left(\frac{\gamma^{\mu}}{a} \partial_{\mu} \psi_{l}^{n, p}\right) \mathcal{F}_{l}^{n, p}+\psi_{r}^{n, p}\left\{\frac{1}{R_{y}}\left(\partial_{4}+2 \frac{a^{\prime}}{a}\right)-i \frac{b}{r_{z}}\left(\partial_{5}+\frac{5 \dot{b}}{2 b}\right)\right\} \mathcal{F}_{r}^{n, p}\right] \otimes \mathcal{S}_{\text {up }} } \\
& +\left[-\left(\frac{\gamma^{\mu}}{a} \partial_{\mu} \psi_{r}^{n, p}\right) \mathcal{F}_{r}^{n, p}+\psi_{l}^{n, p}\left\{\frac{1}{R_{y}}\left(\partial_{4}+2 \frac{a^{\prime}}{a}\right)+i \frac{b}{r_{z}}\left(\partial_{5}+\frac{5 \dot{b}}{2 b}\right)\right\} \mathcal{F}_{l}^{n, p}\right] \otimes \mathcal{S}_{\mathrm{dn}} .
\end{aligned}
$$


Expectedly, the two Weyl fields $\psi_{l / r}^{n, p}$ (at each level) combine to give a Dirac fermion, and this results in

$$
\begin{aligned}
\gamma^{\mu} \partial_{\mu} \psi_{l / r}^{n, p} & =M_{n, p} \psi_{r / l}^{n, p} \\
0 & =\frac{M_{n, p}}{a} \mathcal{F}_{l}^{n, p}+\frac{1}{R_{y}}\left(\partial_{4}+2 \frac{a^{\prime}}{a}\right) \mathcal{F}_{r}^{n, p}-i \frac{b}{r_{z}}\left(\partial_{5}+\frac{5}{2} \frac{\dot{b}}{b}\right) \mathcal{F}_{r}^{n, p} \\
0 & =\frac{-M_{n, p}}{a} \mathcal{F}_{r}^{n, p}+\frac{1}{R_{y}}\left(\partial_{4}+2 \frac{a^{\prime}}{a}\right) \mathcal{F}_{l}^{n, p}+i \frac{b}{r_{z}}\left(\partial_{5}+\frac{5}{2} \frac{\dot{b}}{b}\right) \mathcal{F}_{l}^{n, p}
\end{aligned}
$$

Effecting a separation of variables, we write

$$
\mathcal{F}_{l / r}^{n, p}\left(x_{4}, x_{5}\right)=\left[a\left(x_{4}\right)\right]^{-2}\left[b\left(x_{5}\right)\right]^{-5 / 2} \widetilde{f}_{l / r}^{n, p}\left(x_{4}\right) f_{l / r}^{p}\left(x_{5}\right)
$$

as this particular parametrization not only removes the spin connection terms from the equations of motion, but also effectively isolates the derivative discontinuities in the wavefunctions at the boundaries. This leads to

$$
\begin{aligned}
& \left(\frac{1}{R_{y}} \partial_{4}-i \frac{b}{r_{z}} \partial_{5}\right) \widetilde{f}_{r}^{n, p}\left(x_{4}\right) f_{r}^{p}\left(x_{5}\right)+\frac{M_{n, p}}{a} \widetilde{f}_{l}^{n, p}\left(x_{4}\right) f_{l}^{p}\left(x_{5}\right)=0 \\
& \left(\frac{1}{R_{y}} \partial_{4}+i \frac{b}{r_{z}} \partial_{5}\right) \widetilde{f}_{l}^{n, p}\left(x_{4}\right) f_{l}^{p}\left(x_{5}\right)-\frac{M_{n, p}}{a} \widetilde{f}_{r}^{n, p}\left(x_{4}\right) f_{r}^{p}\left(x_{5}\right)=0 .
\end{aligned}
$$

Clearly, $f_{r / l}^{p}\left(x_{5}\right)=1$ and $\widetilde{f}_{r / l}^{n, p}\left(x_{4}\right)=1$ satisfy the above for $M_{n, p}=0$ and these, if permitted by the boundary conditions, would denote the ground state.

For nonzero $M_{n, p}$, these coupled equations can be diagonalized in a fashion analogous to that for the flat space case, albeit at the cost of introducing slightly more complicated operators, viz.

$$
\begin{aligned}
0 & =\left(a D^{-} a D^{+}+M_{n, p}^{2}\right) \widetilde{f}_{r}^{n, p}\left(x_{4}\right) f_{r}^{p}\left(x_{5}\right) \\
0 & =\left(a D^{+} a D^{-}+M_{n, p}^{2}\right) \widetilde{f}_{l}^{n, p}\left(x_{4}\right) f_{r}^{p}\left(x_{5}\right) \\
D^{ \pm} & \equiv \frac{1}{R_{y}} \partial_{4} \mp i \frac{b}{r_{z}} \partial_{5} .
\end{aligned}
$$

On separating, these yield

$$
\begin{aligned}
& 0=a\left(x_{4}\right) \partial_{4}\left[a\left(x_{4}\right) \partial_{4} \widetilde{f}_{l / r}^{n, p}\left(x_{4}\right)\right]+R_{y}^{2}\left[M_{n p}^{2}-m_{p}^{2} a^{2}\left(x_{4}\right)\right] \widetilde{f}_{l / r}^{n, p}\left(x_{4}\right) \\
& 0=b\left(x_{5}\right) \partial_{5}\left(b\left(x_{5}\right) \partial_{5}+i c \operatorname{sgn}\left(x_{4}\right) \frac{r_{z}}{R_{y}}\right) f_{r}^{p}\left(x_{5}\right)+m_{p}^{2} r_{z}^{2} f_{r}^{p}\left(x_{5}\right) \\
& 0=b\left(x_{5}\right) \partial_{5}\left(b\left(x_{5}\right) \partial_{5}-i c \operatorname{sgn}\left(x_{4}\right) \frac{r_{z}}{R_{y}}\right) f_{l}^{p}\left(x_{5}\right)+m_{p}^{2} r_{z}^{2} f_{l}^{p}\left(x_{5}\right) .
\end{aligned}
$$

Clearly, the $x_{5}$-equations can be factorized and their solutions, in the bulk, would satisfy ${ }^{3}$

$$
b\left(x_{5}\right) \partial_{5} f_{l / r}=\left[i \frac{c r_{z}}{2 R_{y}} \kappa_{l / r}\right] f_{l / r}
$$

\footnotetext{
${ }^{3}$ The ostensible derivative discontinuities which would, putatively, have exchanged $f_{l}$ and $f_{r}$ at the boundary, is actually of no consequence at all as the physical range corresponds to $x_{4} \geq 0$.
} 
where the exponent has been factorized for future convenience. The constants $\kappa_{l / r}$ are solutions of quadratic equations, and are given by

$$
\begin{gathered}
\kappa_{r}^{ \pm}=-1 \pm \sqrt{1+4 \frac{m_{p}^{2} R_{y}^{2}}{c^{2}}} \\
\kappa_{l}^{ \pm}=1 \pm \sqrt{1+4 \frac{m_{p}^{2} R_{y}^{2}}{c^{2}}} .
\end{gathered}
$$

This leads to

$$
\begin{aligned}
f_{l}\left(x_{5}\right) & =d_{l}^{+} \exp \left[i \kappa_{l}^{+} \Theta_{k}\left(x_{5}\right)\right]+d_{l}^{-} \exp \left[i \kappa_{l}^{-} \Theta_{k}\left(x_{5}\right)\right] \\
f_{r}\left(x_{5}\right) & =d_{r}^{+} \exp \left[i \kappa_{r}^{+} \Theta_{k}\left(x_{5}\right)\right]+d_{r}^{-} \exp \left[i \kappa_{r}^{-} \Theta_{k}\left(x_{5}\right)\right] \\
\Theta_{k}\left(x_{5}\right) & \equiv \tan ^{-1}\left(\tanh \frac{k x_{5}}{2}\right) .
\end{aligned}
$$

To determine the constants $d_{l / r}^{ \pm}$, we need to impose the boundary conditions, which, for phenomenological reasons, must be different for each chiral projection. To be specific, for the SU $(2)_{L}$-doublet fields, we impose Neumann conditions for $f_{l}$ and Dirichlet for $f_{R}$. This, later on, would ensure that the zero-mode four-dimensional fermion would be a left-handed field. ${ }^{4}$ In other words, we demand $\left.\partial_{5} f_{l}\right|_{x_{5}=0, \pi}=0$ and $\left.f_{r}\right|_{x_{5}=0, \pi}=0$. Thus,

$$
\begin{aligned}
& \left.\partial_{5} f_{l}\right|_{x_{5}=0}=0 \quad \Longrightarrow \quad d_{l}^{-} \kappa_{l}^{-}=-d_{l}^{+} \kappa_{l}^{+} \\
& \left.\partial_{5} f_{l}\right|_{x_{5}=\pi}=0 \quad \Longrightarrow \quad 0=\kappa_{l}^{-} \sin \left(\left(\kappa_{l}^{-}-1\right) \Theta_{k}(\pi)\right) \text {. }
\end{aligned}
$$

Clearly, the trivial solution $\kappa_{l}^{-}=0$, corresponds to $m_{p}=0$ and $f_{l}\left(x_{5}\right)=1$. Other solutions are given by

$$
\sqrt{1+4 \frac{m_{p}^{2} R_{y}^{2}}{c^{2}}}=\frac{p \pi}{\Theta_{k}(\pi)}
$$

with $p \in \mathbb{Z}^{+}$, thereby quantizing $m_{p}$. Similarly, for $f_{r}$ we have

$$
\begin{aligned}
\left.f_{r}\right|_{x_{5}=0}=0 & \Longrightarrow \quad d_{r}^{+}=-d_{r}^{-} \\
\left.f_{r}\right|_{x_{5}=\pi}=0 & \Longrightarrow \quad 0=\sin \left(\left(\kappa_{r}^{+}+1\right) \Theta_{k}(\pi)\right)
\end{aligned}
$$

leading to (as expected) a mass quantization condition identical to that for the left-chiral fields. Of course, the $m_{p}=0$ state does not exist for the $f_{r}$, implying, in turn, the phenomenologically required condition of there being no massless right-handed $\mathrm{SU}(2)_{L^{-}}$ doublet field.

Given a $m_{p}$, we can now solve the first of eqs. (3.15), to $\widetilde{f}_{l / r}^{n, p}$ in terms of Bessel functions, namely

$$
\begin{aligned}
\widetilde{f}_{l}^{n, p}\left(x_{4}\right) & =e^{c\left|x_{4}\right| / 2}\left[c_{1} J_{\nu_{p}}\left(x_{n p} e^{c\left(\left|x_{4}\right|-\pi\right)}\right)+c_{2} Y_{\nu_{p}}\left(x_{n p} e^{c\left(\left|x_{4}\right|-\pi\right)}\right)\right] \\
\widetilde{f}_{r}^{n, p}\left(x_{4}\right) & =e^{c\left|x_{4}\right| / 2}\left[c_{3} J_{\nu_{p}}\left(x_{n p} e^{c\left(\left|x_{4}\right|-\pi\right)}\right)+c_{4} Y_{\nu_{p}}\left(x_{n p} e^{c\left(\left|x_{4}\right|-\pi\right)}\right)\right] \\
\nu_{p} & \equiv \sqrt{\frac{1}{4}+\frac{m_{p}^{2} R_{y}^{2}}{c^{2}}}=\frac{p \pi}{2 \Theta_{k}(\pi)} \\
x_{n p} & \equiv M_{n p} \frac{R_{y}}{c} e^{c \pi}
\end{aligned}
$$

\footnotetext{
${ }^{4}$ For the $\mathrm{SU}(2)_{L}$-singlets, the condition would be opposite resulting in only the right-handed component having a zero-mode.
} 
where $c_{i}$ s are the constants of integration. It is interesting to note that for $m_{p}=0$, we get back the wave functions corresponding to the five-dimensional RS theory [49]. In particular, the massless mode (i.e., $M_{0,0}=0$ and obtainable only for $m_{0}=0$ ) has, as expected, a flat profile.

\subsection{KK masses for the fermions}

As we shall see in the next section, the issue of spontaneous symmetry breaking is a tricky one, for the inclusion of a bulk Higgs has been shown [43] to resuscitate the hierarchy problem. Localizing the higgs onto a brane obviates the problem, though. Given this, we continue under the assumption of the six-dimensional fields to be strictly massless and, thus, the only contribution to the masses of the four-dimensional components would be those due to the compactification. Incorporating the effect of the Higgs field is a straightforward exercise, and would be undertaken in section 5. In any case, with the compactification scale being much larger than the electroweak scale, the Higgs contribution would be of little importance to any but the lowest mode and can be treated as a perturbation.

Determining the spectrum, as usual, needs the imposition of the boundary conditions. While we already have done so for the $x_{5}$-modes, the $x_{4}$-component is still unrestricted. Noting that the orbifolding demands that the fermion wavefunctions be even in the $x_{4^{-}}$ direction, these must satisfy

$$
\left.\partial_{4} \tilde{f}_{l}^{n, p}\left(x_{4}\right)\right|_{x_{4}=0}=0,\left.\quad \partial_{4} \tilde{f}_{r}^{n, p}\left(x_{4}\right)\right|_{x_{4}=\pi}=0 .
$$

Rather than attempt to solve for the above for the most general choice of $c$ and $k$, we restrict ourselves to the two cases that are of relevance in resolving the hierarchy problem, viz. small $k$ (large $c$ ) on the one hand and large $k$ (small $c$ ) on the other.

\subsubsection{Small $k$ and large $c$}

The aforementioned boundary conditions, respectively, give

$$
\frac{-c_{2}}{c_{1}}=\frac{e^{-c \pi} x_{n p}\left[J_{\nu_{p}-1}\left(x_{n p} e^{-c \pi}\right)-J_{\nu_{p}+1}\left(x_{n p} e^{-c \pi}\right)\right]+J_{\nu_{p}}\left(x_{n p} e^{-c \pi}\right)}{e^{-c \pi} x_{n p}\left[Y_{\nu_{p}-1}\left(x_{n p} e^{-c \pi}\right)-Y_{\nu_{p}+1}\left(x_{n p} e^{-c \pi}\right)\right]+Y_{\nu_{p}}\left(x_{n p} e^{-c \pi}\right)}
$$

and

$$
\frac{-c_{2}}{c_{1}}=\frac{x_{n p}\left[J_{\nu_{p}-1}\left(x_{n p}\right)-J_{\nu_{p}+1}\left(x_{n p}\right)\right]+J_{\nu_{p}}\left(x_{n p}\right)}{x_{n p}\left[Y_{\nu_{p}-1}\left(x_{n p}\right)-Y_{\nu_{p}+1}\left(x_{n p}\right)\right]+Y_{\nu_{p}}\left(x_{n p}\right)} .
$$

As $e^{-c \pi}$ is negligibly small in this regime, the corresponding $Y_{\nu}$ are very large. Consequently, in this regime, the two equations above are simultaneously satisfied only if

$$
x_{n p}\left[J_{\nu_{p}-1}\left(x_{n p}\right)-J_{\nu_{p}+1}\left(x_{n p}\right)\right]+J_{\nu_{p}}\left(x_{n p}\right)=0,
$$

thereby determining the quantized values for $x_{n p}$ (for a given $\nu_{p}$ determined, in turn, by eqs. (3.19) \& (3.18)). For a particular choice of the parameters, these are exhibited in figure 2.

For the right-chiral fields, relations analogous to eqs. (3.21) \& (3.22) would obtain. It is easy to see that the corresponding set of $x_{n p}$ s identically match those in eq. (3.23), but for the zero mode. 

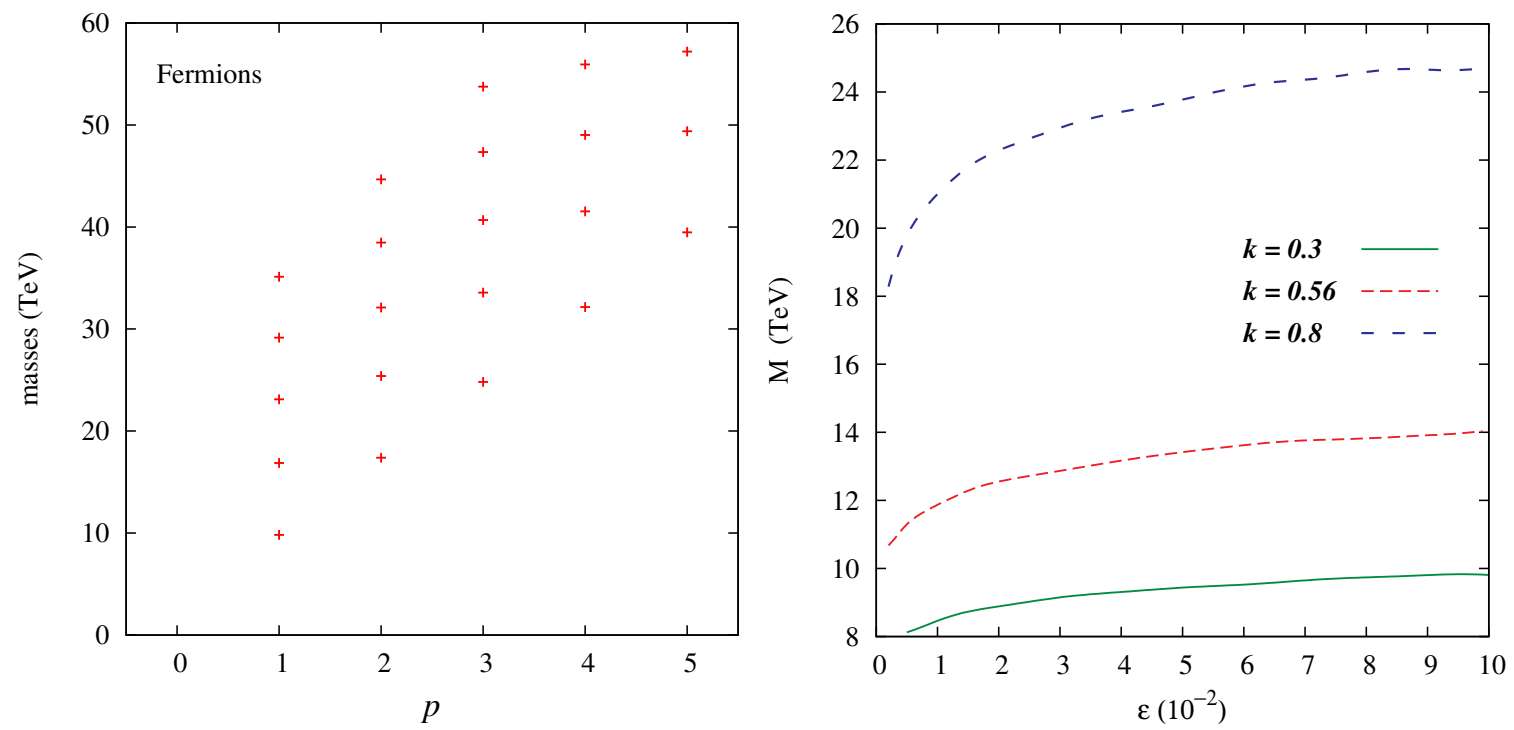

Figure 2. (Left panel) A sample Kaluza-Klein spectrum that satisfies eq. (3.23) originating from a six-dimensional chiral fermion, for $k=0.3, \alpha=49$ and $\epsilon=0.0775$ (see eq. (2.3)). Only the first five $n$ levels corresponding to each $p$ are shown. (Right panel) The dependence of the mass of the lowest KK mode on $\epsilon$. In both the panels, $R_{y}$ set to satisfy the hierarchy eq. (2.7).

\subsubsection{Large $k$ and small $c$}

Since $c$ is now almost infinitesimally small, $a\left(x_{4}\right) \approx 1$ is a very good approximation and eq. (3.14) can be simplified down to

$$
\partial_{4}^{2} \widetilde{f}_{l / r}^{n, p}\left(x_{4}\right)=-\left(M_{n p}^{2}-m_{p}^{2}\right) R_{y}^{2} \widetilde{f}_{l / r}^{n, p}\left(x_{4}\right) .
$$

This, of course, yields plane wave solutions, which is as expected since such a small warping means that the space is essentially flat. Hence, the spectrum is given by

$$
M_{n p}^{2} \approx \frac{n^{2}}{R_{y}^{2}}+m_{p}^{2}
$$

As $R_{y}$ is very small, we could as well neglect the $n \neq 0$ modes in any discussion of $\mathrm{TeV}$ scale physics. In other words, for all practical purposes, the fermions act as if they are confined onto a brane.

\section{The gauge bosons}

To begin with, we examine the mass spectrum of the gauge bosons postponing discussion of all interactions until later. Hence, it is convenient to consider only a U(1) theory in the six dimensional bulk. Similarly, rather than concerning ourselves with the issue of symmetry breaking in the bulk, we would introduce an explicit mass term so as to understand the consequences of a bulk mass term.

The gauge boson Lagrangian is given by

$$
\mathcal{L}=\frac{-1}{4} \sqrt{-g} F_{M N} F^{M N}+\mathcal{L}_{g f}
$$


While the structure of the gauge-fixing term is, a priori, undetermined, it is often useful to restrict ourselves to a covariant choice, so as to manifestly respect the symmetries of the theory, which, at first sight, would seem to be diffeomorphism invariance. Note, however, that the presence of the boundary branes (and the orbifolding), actually reduces the symmetry away from the bulk to only a four-dimensional general coordinate invariance. Hence, we introduce a curved-space analog of the oft-used (at least in the case of flat extra dimensions [50]) generalized $R_{\zeta}$ gauge which, while respecting the four-dimensional general coordinate invariance, also serves to eliminate the cumbersome kinetic mixing terms between $A_{\mu}$ and $A_{4,5}$. To be specific, we have

$$
\begin{aligned}
\mathcal{L}_{g f} & =\frac{-\sqrt{-g}}{2 \zeta}\left[g^{\mu \nu}\left\{\partial_{\mu} A_{\nu}-\frac{\zeta}{2}\left(\Gamma_{\mu \nu}^{4} A_{4}+\Gamma_{\mu \nu}^{5} A_{5}\right)\right\}+\zeta\left(g^{44} D_{4} A_{4}+g^{55} D_{5} A_{5}\right)\right]^{2} \\
& =\frac{-R_{y} r_{z} b}{2 \zeta}\left[\eta^{\mu \nu} \partial_{\mu} A_{\nu}+\frac{\zeta}{b}\left(\partial_{4} \frac{a^{2} b A_{4}}{R_{y}^{2}}+\partial_{5} \frac{a^{2} b^{3} A_{5}}{r_{z}^{2}}\right)\right]^{2} .
\end{aligned}
$$

This does not exhaust all of the gauge symmetries. On compactification down to four dimensions, the components $A_{4,5}$ would, naturally, give rise to a 'tower of towers' of scalars transforming under the adjoint representation of the gauge group. On the other hand, the very act of the higher modes of $A_{\mu}$ becoming massive could be viewed as the result of a Higgs mechanism wherein the adjoint Goldstone has been absorbed. In other words, in the unitary gauge, only one linear combination of $A_{4,5}$ may survive. But as in the case with UED, this conclusion is dependent on the gauge choice. In particular, for $\zeta=1$, both sets of the scalars survive. Adopting, for the time, the Minkowski metric, the quadratic term for the vector field is now given by

$$
\mathcal{L}_{A_{\mu}}=\frac{-R_{y} r_{z}}{2}\left[b A_{\kappa}\left(-\partial^{2} \eta^{\kappa \lambda}+\partial^{\lambda} \partial^{\kappa}\right) A_{\lambda}+\frac{a^{2} b}{R_{y}^{2}}\left(\partial_{4} A_{\kappa}\right)\left(\partial_{4} A^{\kappa}\right)+\frac{a^{2} b^{3}}{r_{z}^{2}}\left(\partial_{5} A_{\kappa}\right)\left(\partial_{5} A^{\kappa}\right)\right],
$$

while for the adjoint scalars, with field redefinitions

$$
\tilde{A}_{4} \equiv \sqrt{\frac{r_{z}}{R_{y}}} A_{4}, \quad \text { and } \quad \tilde{A}_{5} \equiv \sqrt{\frac{R_{y}}{r_{z}}} A_{5},
$$

it is

$$
\begin{aligned}
& \mathcal{L}_{\tilde{A}_{4}}=\frac{-1}{2}\left[a^{2} b\left(\partial_{\mu} \tilde{A}_{4}\right)\left(\partial^{\mu} \tilde{A}_{4}\right)+\frac{a^{4} b^{3}}{r_{z}^{2}}\left(\partial_{5} \tilde{A}_{4}\right)^{2}+\frac{1}{R_{y}^{2} b}\left\{\partial_{4}\left(a^{2} b \tilde{A}_{4}\right)\right\}^{2}\right] \\
& \mathcal{L}_{\tilde{A}_{5}}=\frac{-1}{2}\left[a^{2} b^{3}\left(\partial_{\mu} \tilde{A}_{5}\right)\left(\partial^{\mu} \tilde{A}_{5}\right)+\frac{a^{4} b^{3}}{R_{y}^{2}}\left(\partial_{4} \tilde{A}_{5}\right)^{2}+\frac{1}{r_{z}^{2} b}\left\{\partial_{5}\left(a^{2} b^{3} \tilde{A}_{5}\right)\right\}^{2}\right]
\end{aligned}
$$

and reminiscent of the action for a scalar field. There also exists a mixing term

$$
\mathcal{L}_{\text {mix }}=2 \frac{1}{R_{y} r_{z}} a^{3} a^{\prime} \tilde{A}_{4} \partial_{5}\left(b^{3} \tilde{A}_{5}\right)
$$

and, hence, ideally, one should rediagonalize the $\tilde{A}_{4}-\tilde{A}_{5}$ system. However, as a zeroth approximation, one may neglect $\mathcal{L}_{\text {mix }}$ altogether and derive the wavefunctions and propagators from $\mathcal{L}_{\tilde{A}_{4}}$ and $\mathcal{L}_{\tilde{A}_{5}}$ alone. Using the thus derived wavefunctions in $\mathcal{L}_{\text {mix }}$, it can 
be seen that this term is numerically rather subdominant. Allied with the fact that the zero modes of $\tilde{A}_{4,5}$ would not survive and only the heavy KK-modes come into play, the neglect of $\mathcal{L}_{\text {mix }}$ has almost no discernible consequence as far as low energy phenomenology is concerned. We shall, thus, continue with this approximation, while bearing in mind that $\mathcal{L}_{\text {mix }}$ ought to be taken into account when dealing with subleading effects as also in the context of questions such as unitarity.

To the quadratic kinetic terms, we may add a mass term (presumably originating from spontaneous symmetry breaking in the bulk), viz.

$$
\mathcal{L}_{\mathcal{M}}=-\frac{\sqrt{-g}}{2} M^{2} A_{N} A^{N}
$$

We may now express the field in terms of the eigenstates of the extra-dimensional parts of the aforementioned differential operators. These are but the analogues of the "plane wave" solutions and given by

$$
\begin{aligned}
& A_{\kappa}=\frac{1}{\sqrt{R_{y} r_{z}}} \sum_{n, p} A_{\kappa}^{(n, p)}\left(x^{\mu}\right) \eta_{n, p}\left(x_{4}\right) \chi_{p}\left(x_{5}\right) \\
& \tilde{A}_{4}=\sum_{n, p} \tilde{A}_{(4)}^{(n, p)}\left(x_{\mu}\right) \eta_{n, p}^{(4)}\left(x_{4}\right) \chi_{p}^{(4)}\left(x_{5}\right) \\
& \tilde{A}_{5}=\sum_{n, p} \tilde{A}_{(5)}^{(n, p)}\left(x_{\mu}\right) \eta_{n, p}^{(5)}\left(x_{4}\right) \chi_{p}^{(5)}\left(x_{5}\right)
\end{aligned}
$$

with the components satisfying the orthogonality relations

$$
\begin{aligned}
\int d x_{5} b\left(x_{5}\right) \chi_{p}\left(x_{5}\right) \chi_{p^{\prime}}\left(x_{5}\right) & =\delta_{p p^{\prime}} \\
\int d x_{4} \eta_{n, p}\left(x_{4}\right) \eta_{n^{\prime}, p^{\prime}}\left(x_{4}\right) & =\delta_{n n^{\prime}} \delta_{p p^{\prime}} \\
\int d x_{5} b\left(x_{5}\right) \chi_{p}^{(4)}\left(x_{5}\right) \chi_{p^{\prime}}^{(4)}\left(x_{5}\right) & =\delta_{p p^{\prime}} \\
\int d x_{4} a^{2}\left(x_{4}\right) \eta_{n, p}^{(4)}\left(x_{4}\right) \eta_{n^{\prime}, p^{\prime}}^{(4)}\left(x_{4}\right) & =\delta_{n n^{\prime}} \delta_{p p^{\prime}} \\
\int d x_{5} b^{3}\left(x_{5}\right) \chi_{p}^{(5)}\left(x_{5}\right) \chi_{p^{\prime}}^{(5)}\left(x_{5}\right) & =\delta_{p p^{\prime}} \\
\int d x_{4} a^{2}\left(x_{4}\right) \eta_{n, p}^{(5)}\left(x_{4}\right) \eta_{n^{\prime}, p^{\prime}}^{(5)}\left(x_{4}\right) & =\delta_{n n^{\prime}} \delta_{p p^{\prime}}
\end{aligned}
$$

The corresponding equations of motion for the vector modes are

$$
\begin{aligned}
\frac{1}{r_{z}^{2}} \partial_{5}\left(b^{3} \partial_{5} \chi_{p}\right)-M^{2} b^{3} \chi_{p} & =-m_{p}^{2} b \chi_{p} \\
\frac{1}{R_{y}^{2}} \partial_{4}\left(a^{2} \partial_{4} \eta_{n, p}\right)-m_{p}^{2} a^{2} \eta_{n, p} & =-m_{n p}^{2} \eta_{n, p},
\end{aligned}
$$


whose solutions could be written as

$$
\begin{aligned}
\chi_{p}\left(x_{5}\right) & =\frac{1}{\bar{B}_{p}} \operatorname{sech}^{3 / 2}\left(k x_{5}\right)\left(c_{1} P_{\nu_{p}}^{u / 2}\left(\tanh k x_{5}\right)+c_{2} Q_{\nu_{p}}^{u / 2}\left(\tanh k x_{5}\right)\right) \\
\eta_{n, p}\left(x_{4}\right) & =\frac{e^{c\left|x_{4}\right|}}{B_{n p}}\left(J_{\nu_{n}}\left(y_{n}\right)+c_{n p} Y_{\nu_{n}}\left(y_{n}\right)\right) \\
y_{n} & \equiv m_{n p} \frac{r_{z}}{k} e^{c\left|x_{4}\right|} \cosh (k \pi)=m_{n p} \frac{R_{y}}{c} e^{c\left|x_{4}\right|} \\
\nu_{n} & =\sqrt{1+\frac{r_{z}^{2}}{k} m_{p}^{2} \cosh ^{2}(k \pi)} \\
\nu_{p} & =\frac{-1}{2}+\nu_{n} \\
u & =\sqrt{9+\frac{4 M^{2} r_{z}^{2}}{k^{2}}}
\end{aligned}
$$

where $c_{1,2}$ (we explicitly retain both as this is useful in studying the boundary conditions) and $c_{n p}$ are arbitrary constants while $\bar{B}_{p}$ and $B_{n p}$ provide the normalization. The associate Legendre functions, appearing also in the description of the fermions, or at any stage of the six-dimensional theory, are reminiscent of the $x_{5}$-dependence of the graviton wavefunctions [32] and are a feature of the nested warping.

The equations of motion for the adjoint scalar $A_{4}$ are

$$
\begin{aligned}
\frac{1}{r_{z}^{2}} \partial_{5}\left(b^{3} \partial_{5}\left(\chi_{p}^{(4)}\right)\right)-M^{2} b^{3} \chi_{p}^{(4)} & =-\tilde{m}_{p}^{2} b \chi_{p}^{(4)} \\
\frac{1}{R_{y}^{2}} \partial_{4}\left(\partial_{4} a^{2} \eta_{n, p}^{(4)}\right)-a^{2} \tilde{m}_{p}^{2} \eta_{n, p}^{(4)} & =-\tilde{m}_{n p}^{2} \eta_{n, p}^{(4)},
\end{aligned}
$$

leading to

$$
\begin{aligned}
& \chi_{p}^{(4)}\left(x_{5}\right)=\frac{1}{\overline{\mathcal{E}}_{p}} \operatorname{sech}^{3 / 2}\left(k x_{5}\right)\left[s_{1} P_{\tilde{\nu}_{p}}^{\tilde{v} / 2}\left(\tanh k x_{5}\right)+s_{2} Q_{\tilde{\nu}_{p}}^{\tilde{v} / 2}\left(\tanh k x_{5}\right)\right] \\
& \eta_{n, p}^{(4)}\left(x_{4}\right)=\frac{1}{\mathcal{E}_{n p}} e^{2 c\left|x_{4}\right|}\left[J_{\tilde{\nu}_{n}}\left(y_{n}\right)+s_{n p} Y_{\tilde{\nu}_{n}}\left(y_{n}\right)\right] \\
& \tilde{\nu}_{n}=\sqrt{\frac{r_{z}^{2}}{k^{2}} \tilde{m}_{p}^{2} \cosh ^{2}(k \pi)} \\
& \tilde{\nu}_{p}=\frac{-1}{2}+\sqrt{1+\frac{r_{z}^{2}}{k^{2}} \tilde{m}_{p}^{2} \cosh ^{2}(k \pi)}=\nu_{p} \\
& \tilde{v}=\sqrt{9+\frac{4 M^{2} r_{z}^{2}}{k^{2}}}=u \text {. }
\end{aligned}
$$

where $s_{1,2}$ and $s_{n p}$ are constants of integration, while $\mathcal{E}_{n p}$ and $\mathcal{E}_{p}$ serve to normalize. Similarly, the equations of motion for the adjoint scalar $A_{5}$ are seen to be

$$
\begin{aligned}
\frac{1}{r_{z}^{2}} \partial_{5}\left(b^{-1} \partial_{5}\left(b^{3} \chi_{p}^{(5)}\right)\right)-M^{2} b^{2} \chi_{p}^{(5)} & =-\bar{m}_{p}^{2} \chi_{p}^{(5)} \\
\frac{1}{R_{y}^{2}} \partial_{4}\left(a^{4} \partial_{4} \eta_{n, p}^{(5)}\right)-a^{4} \bar{m}_{p}^{2} \eta_{n, p}^{(5)} & =-\bar{m}_{n p}^{2} a^{2} \eta_{n, p}^{(5)},
\end{aligned}
$$


leading to

$$
\begin{aligned}
\chi_{p}^{(5)}\left(x_{5}\right) & =\frac{1}{\overline{\mathcal{D}}_{p}} \operatorname{sech}^{5 / 2}\left(k x_{5}\right)\left[d_{1} P_{\bar{\nu}_{p}}^{\bar{v} / 2}\left(\tanh k x_{5}\right)+d_{2} Q_{\bar{\nu}_{p}}^{\bar{v} / 2}\left(\tanh k x_{5}\right)\right] \\
\eta_{n, p}^{(5)}\left(x_{4}\right) & =\frac{1}{\mathcal{D}_{n p}} e^{2 c\left|x_{4}\right|}\left[J_{\bar{\nu}_{n}}\left(y_{n}\right)+d_{n p} Y_{\bar{\nu}_{n}}\left(y_{n}\right)\right] \\
\bar{\nu}_{n} & =\sqrt{4+\frac{r_{z}^{2}}{k^{2}} \bar{m}_{p}^{2} \cosh ^{2}(k \pi)} \\
\bar{\nu}_{p} & =\frac{-1}{2}+\sqrt{1+\frac{r_{z}^{2}}{k^{2}} \bar{m}_{p}^{2} \cosh ^{2}(k \pi)}=\nu_{p} \\
\bar{v} & =\sqrt{1+\frac{4 M^{2} r_{z}^{2}}{k^{2}}} .
\end{aligned}
$$

Once again, $d_{1,2}$ and $d_{n p}$ are constants of integration, while $\overline{\mathcal{D}}_{p}$ and $\mathcal{D}_{n p}$ provide normalizations. With these conditions in place, the quadratic part of the Lagrangian for the vector fields can be expressed in terms of the KK-towers as

$$
\mathcal{L}_{A_{\mu}}=\sum_{n, p}\left[\frac{-1}{4} F_{\mu \nu}^{(n, p)} F^{\mu \nu(n, p)}-\frac{1}{2} m_{n p}^{2} A_{\mu}^{(n, p)} A^{\mu(n, p)}-\frac{1}{2}\left(\partial_{\mu} A^{\mu(n, p)}\right)^{2}\right],
$$

while, for the adjoint scalars, we have

$$
\mathcal{L}_{A_{5}}=-\frac{1}{2}\left(\partial_{\mu} \tilde{A}_{4}^{(n, p)}\right)^{2}-\frac{1}{2} \tilde{m}_{n p}^{2} \tilde{A}_{4}^{(n, p) 2},
$$

and

$$
\mathcal{L}_{A_{5}}=-\frac{1}{2}\left(\partial_{\mu} \tilde{A}_{5}^{(n, p)}\right)^{2}-\frac{1}{2} \bar{m}_{n p}^{2} \tilde{A}_{5}^{(n, p) 2}
$$

\subsection{Gauge and adjoint scalar masses}

Our aim, now, is to compute the allowed values for $m_{n p}, \tilde{m}_{n p}$ and $\bar{m}_{n p}$. In each case, one must first find the $x_{5}$-equation eigenvalues $\left(m_{p}, \tilde{m}_{p}\right.$ and $\bar{m}_{p}$ respectively) using the boundary conditions for the corresponding wavefunctions and, then, find the desired spectrum in terms of these. For ease of appreciation, we perform the exercise in the reverse order. We first establish the general conditions and then examine the situation for the two particular cases of interest (in terms of the relative sizes of $k$ and $c$ ).

In doing so, it should be borne in mind that the bulk mass term $M$, that we have considered until now, would identically disappear, to be replaced, in the electroweak sector, by the spontaneous breaking term. The latter, in all our constructions, would be confined to a brane, and its effective scale would, naturally, turn out to be the electroweak scale to be compared with the much larger compactification scales $R_{y}^{-1}$ or $r_{z}^{-1}$. Thus, it stands to reason that the effect of the spontaneous breaking term in the spectrum and the wavefunctions would be negligible, except, perhaps, for the ground state. Indeed, as the experience with the RS case [56] has shown, its role there too is subdominant. Consequently, we will postpone a discussion of such terms until section 4.1.5. 


\subsubsection{Boundary conditions in the $x_{4}$-direction}

As of now, we have not considered the existence of any localized fields. ${ }^{5}$ Consequently, the wavefunctions $\eta_{n, p}\left(x_{4}\right)$ must be differentiable, especially at the ends of the world. This implies that

$$
-c_{n p}=\frac{\alpha_{n p} e^{c\left(\left|x_{4}\right|-\pi\right)} J_{\nu_{p}-\frac{1}{2}}\left(\alpha_{n p} e^{c\left(\left|x_{4}\right|-\pi\right)}\right)-\left(-\frac{1}{2}+\nu_{p}\right) J_{\nu_{p}+\frac{1}{2}}\left(\alpha_{n p} e^{c\left(\left|x_{4}\right|-\pi\right)}\right)}{\alpha_{n p} e^{c\left(\left|x_{4}\right|-\pi\right)} Y_{\nu_{p}-\frac{1}{2}}\left(\alpha_{n p} e^{c\left(\left|x_{4}\right|-\pi\right)}\right)-\left(-\frac{1}{2}+\nu_{p}\right) Y_{\nu_{p}+\frac{1}{2}}\left(\alpha_{n p} e^{c\left(\left|x_{4}\right|-\pi\right)}\right)}
$$

for each of $x_{4}=0, \pi$. Here, we have defined

$$
\alpha_{n p} \equiv m_{n p} \frac{R_{y}}{c} e^{c \pi} .
$$

Once $\nu_{p}$ is known, the two conditions of eq. (4.17), together, determine $\eta_{n, p}\left(x_{4}\right)$ as well as serve to quantize $\alpha_{n p}$ (and, hence, $m_{n p}$ ).

Similarly, since $a\left(x_{4}\right)^{2} \eta_{n, p}^{(4)}\left(x_{4}\right)$ and $\eta_{n, p}^{(5)}\left(x_{4}\right)$ are even functions, their derivatives have to vanish at both the fixed points $\left(x_{4}=0, \pi\right)$ and this gives

$$
s_{n p}=-\left.\frac{J_{\tilde{\nu}_{n}-1}\left(\tilde{\alpha}_{n p} e^{c\left(\left|x_{4}\right|-\pi\right)}\right)-J_{\tilde{\nu}_{n}+1}\left(\tilde{\alpha}_{n p} e^{c\left(\left|x_{4}\right|-\pi\right)}\right)}{Y_{\tilde{\nu}_{n}-1}\left(\tilde{\alpha}_{n p} e^{c\left(\left|x_{4}\right|-\pi\right)}\right)-Y_{\tilde{\nu}_{n}+1}\left(\tilde{\alpha}_{n p} e^{c\left(\left|x_{4}\right|-\pi\right)}\right)}\right|_{x_{4}=0, \pi}
$$

and

$$
d_{n p}=-\left.\frac{\bar{\alpha}_{n p} e^{c\left(\left|x_{4}\right|-\pi\right)} J_{\bar{\nu}_{n}+1}\left(\bar{\alpha}_{n p} e^{c\left(\left|x_{4}\right|-\pi\right)}\right)-\left(2+\bar{\nu}_{n}\right) J_{\bar{\nu}_{n}}\left(\bar{\alpha}_{n p} e^{c\left(\left|x_{4}\right|-\pi\right)}\right)}{\bar{\alpha}_{n p} e^{c\left(\left|x_{4}\right|-\pi\right)} Y_{\bar{\nu}_{n}+1}\left(\bar{\alpha}_{n p} e^{c\left(\left|x_{4}\right|-\pi\right)}\right)-\left(2+\bar{\nu}_{n}\right) Y_{\bar{\nu}_{n}}\left(\bar{\alpha}_{n p} e^{c\left(\left|x_{4}\right|-\pi\right)}\right)}\right|_{x_{4}=0, \pi}
$$

for $\eta_{n, p}^{(4)}$ and $\eta_{n, p}^{(5)}$ respectively. Here,

$$
\tilde{\alpha}_{n p}=\tilde{m}_{n p} \frac{R_{y}}{c} e^{c \pi} \quad \text { and } \quad \bar{\alpha}_{n p}=\bar{m}_{n p} \frac{R_{y}}{c} e^{c \pi} .
$$

Again, once $\tilde{\nu}_{n}$ and $\bar{\nu}_{n}$ is known, the two conditions in eq. (4.19) and eq. (4.20) together determine $\eta_{n, p}^{(4,5)}\left(x_{4}\right)$ as well as quantize the masses.

\subsubsection{Boundary conditions in the $x_{5}$-direction}

As $\chi_{p}\left(x_{5}\right)$ are even functions of $x_{5}$, their derivatives would vanish at $x_{5}=0$. This translates to

$$
\cot \theta_{p} \equiv \frac{c_{1}}{c_{2}}=\frac{-\pi}{2} \cot \frac{\pi\left(\nu_{p}+u / 2\right)}{2} .
$$

An analogous condition would be obtained for the derivative at $x_{5}=\pi$, but that is best analyzed separately for small and large $k$, which we come to later.

We have already seen that since $\eta_{n, p}^{(4,5)}\left(x_{4}\right)$ are even functions, they need to satisfy Neumann boundary conditions at $x_{4}=0, \pi$. Thus, the absence of massless adjoint scalars

\footnotetext{
${ }^{5}$ The situation would change when we introduce the Higgs field and we shall explicitly take this into consideration in section 5 .
} 
necessitates that we impose Dirichlet boundary condition (a consequence of the orbifolding) on $\chi_{p}^{(4,5)}\left(x_{5}\right)$, namely $\chi_{p}^{(4,5)}(0)=0=\chi_{p}^{(4,5)}(\pi)$. This, then, implies that

$$
\frac{-s_{2}}{s_{1}}=\frac{P_{\tilde{\nu}_{p}}^{\tilde{v} / 2}(0)}{Q_{\tilde{\nu}_{p}}^{\tilde{v} / 2}(0)}=\frac{P_{\tilde{\nu}_{p}}^{\tilde{v} / 2}(\tanh k \pi)}{Q_{\tilde{\nu} p}^{\tilde{v} / 2}(\tanh k \pi)}, \frac{-d_{2}}{d_{1}}=\frac{P_{\bar{\nu}_{p}}^{\bar{v} / 2}(0)}{Q_{\bar{\nu}_{p}}^{\bar{v} / 2}(0)}=\frac{P_{\bar{\nu}_{p} / 2}^{\bar{v} / 2}(\tanh k \pi)}{Q_{\bar{\nu}_{p}}^{\bar{v} / 2}(\tanh k \pi)} .
$$

In other words, the eigenvalue spectrum is given by

$$
P_{\tilde{\nu}_{p}}^{\tilde{v} / 2}(0) Q_{\tilde{\nu}_{p}}^{\tilde{v} / 2}(\tanh k \pi)-Q_{\tilde{\nu}_{p}}^{\tilde{v} / 2}(0) P_{\tilde{\nu}_{p}}^{\tilde{v} / 2}(\tanh k \pi)=0
$$

for $A_{4}$ and

$$
P_{\bar{\nu}_{p}}^{\bar{v} / 2}(0) Q_{\bar{\nu}_{p}}^{\bar{v} / 2}(\tanh k \pi)-Q_{\bar{\nu}_{p}}^{\bar{v} / 2}(0) P_{\bar{\nu}_{p}}^{\bar{v} / 2}(\tanh k \pi)=0
$$

for $A_{5}$.

At this point, it is worthwhile to remember our earlier discussion about the limit of vanishing bulk mass $(M=0)$. This immediately leads to $\bar{v}=1$ in eqs. (4.16). Now, if we look for $\bar{m}_{p}=0$ (for the $\tilde{A}_{5}$ spectrum), then we need to concentrate on $\bar{\nu}_{p}=1 / 2$ in eq. (4.24) above, or,

$$
P_{1 / 2}^{1 / 2}(0) Q_{1 / 2}^{1 / 2}(\tanh k \pi)-Q_{1 / 2}^{1 / 2}(0) P_{1 / 2}^{1 / 2}(\tanh k \pi)=0 .
$$

However, since the function $P_{1 / 2}^{1 / 2}(x) / Q_{1 / 2}^{1 / 2}(x)$ is monotonic, this equation can never be satisfied for any $k$. In other words, $\bar{m}_{p}=0$ is strictly disallowed. Equivalently, not only is the unwanted zero mode $A_{5}^{(0,0)}\left(x_{\mu}\right)$ absent, but all the modes $\tilde{A}_{5}^{(n, 0)}\left(x_{\mu}\right)$ do not exist. ${ }^{6}$

Similarly, for the $\tilde{A}_{4}$ spectrum, $M=0$ would imply $\tilde{v}=3$, leading to the $Q_{1 / 2}^{\tilde{v} / 2}$ vanishing identically. The imposition of the aforementioned boundary condition then implies that the corresponding $\chi_{0}^{(4)}\left(x_{5}\right)$ must vanish identically too. Thus, once again, the requirement that there be no massless $\chi^{(4)}$ scalar has the consequence that the entire putative tower comprising of the modes $\tilde{A}_{4}^{(n, 0)}\left(x_{\mu}\right)$ disappears identically.

For both cases, the argument is quite robust and carries through even in the presence of brane-localized spontaneous symmetry breaking term.

As for the rest of the spectrum, this, along with that for the gauge boson excitations, is best analyzed separately for large and small $k$, and this we come to next.

\subsubsection{Small $k$ and large $c$}

Since the Legendre functions are well-behaved in this domain, we could use the Neumann boundary conditions for $\chi_{p}\left(x_{5}\right)$ at $x_{5}=\pi$ in a straightforward fashion, and this implies

$$
\begin{aligned}
0= & \cot \theta_{p}\left(1-2 \nu_{p}\right) \tau_{\pi} P_{\nu_{p}}^{u / 2}\left(\tau_{\pi}\right)+\cot \theta_{p}\left(2+2 \nu_{p}-u\right) P_{\nu_{p}+1}^{u / 2}\left(\tau_{\pi}\right) \\
& +\left(1-2 \nu_{p}\right) \tau_{\pi} Q_{\nu_{p}}^{u / 2}\left(\tau_{\pi}\right)+\left(2+2 \nu_{p}-u\right) Q_{\nu_{p}+1}^{u / 2}\left(\tau_{\pi}\right)
\end{aligned}
$$

where $\tau_{\pi} \equiv \tanh (k \pi)$. This equation has to be solved numerically to obtain the discrete set of values allowed to $\nu_{p}$.

\footnotetext{
${ }^{6}$ This is reminiscent of the fermion spectrum.
} 

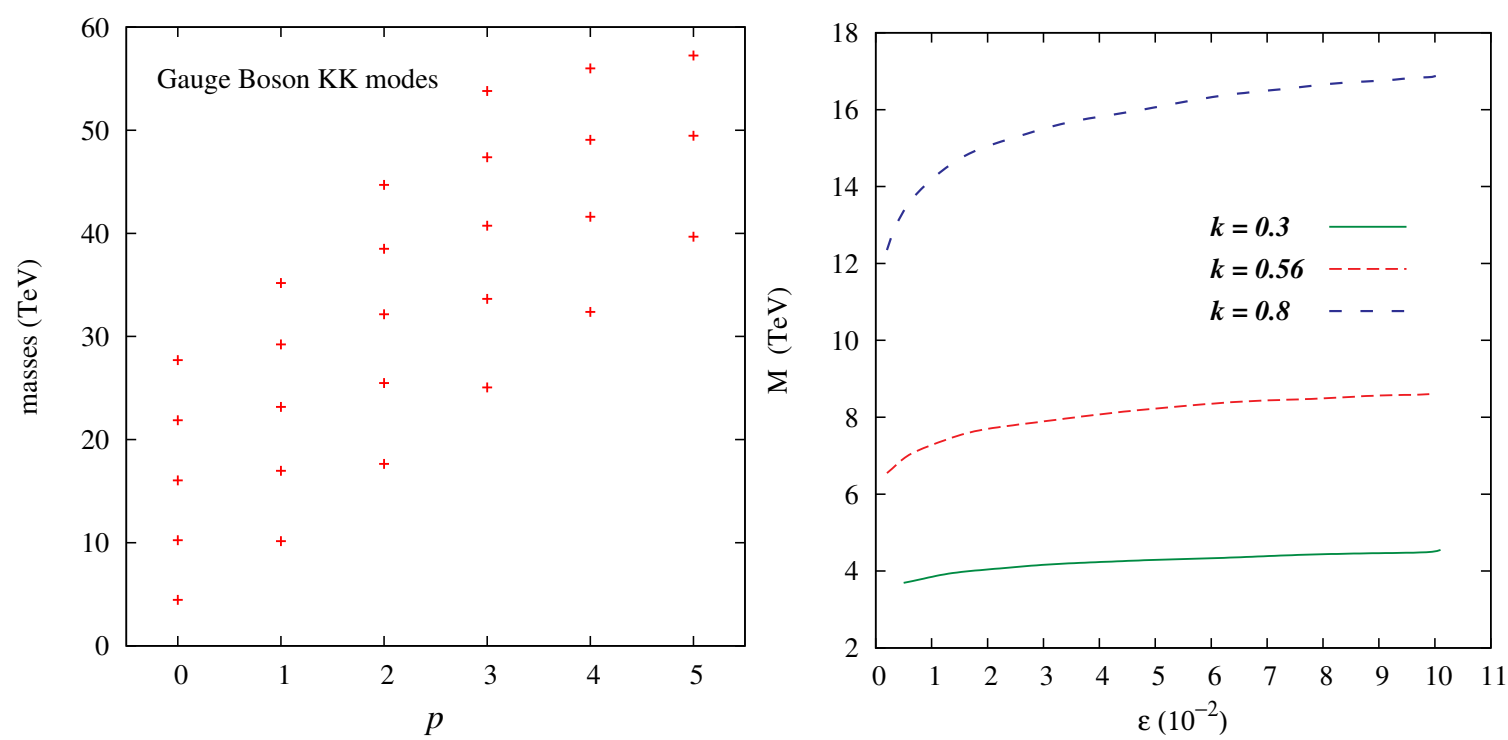

Figure 3. (Left panel)The spectrum of vector gauge bosons with zero bulk mass for $\mathrm{k}=0.3, \alpha=49$ and $\epsilon=0.0775$. Only the first five $n$ levels corresponding to each $p$ are shown. (Right panel) The dependence of the mass of the lowest KK mode on $\epsilon$. In both the panels, $R_{y}$ set to satisfy the hierarchy eq. (2.7).

For a given $\nu_{p}$, to solve for $m_{n p}$, we need to consider both of eqs. (4.17). Note that, for the $(k, c)$ values under discussion, the combination $R_{y}^{-1} e^{-c \pi}$ roughly gives the electroweak scale [32]. Thus, if $m_{n p}$ are to be important in low-energy phenomenology, $\alpha_{n p} \lesssim \mathcal{O}(1)$. On the other hand, with $e^{c \pi}$ being very large, the argument of the Bessel functions essentially vanishes at $x_{4}=0$. This implies that $c_{n p} \approx 0$, and using this in the boundary condition at $x_{4}=\pi$, we have

$$
2 \alpha_{n p} J_{\nu_{p}-1 / 2}\left(\alpha_{n p}\right)+\left(1-2 \nu_{p}\right) J_{\nu_{p}+1 / 2}\left(\alpha_{n p}\right)=0 .
$$

Solving this, for a given $\nu_{p}$, would lead to the gauge boson KK tower starting with $A_{\mu}^{(1,0)}$.

Let us, now, turn our attention to the adjoint scalars. Quite similar to the case for the vector modes, the two eqs. (4.19) are satisfied only if $s_{n p}=0$ and, hence,

$$
J_{\tilde{\nu}_{n}-1}\left(\tilde{\alpha}_{n p}\right)-J_{\tilde{\nu}_{n}+1}\left(\tilde{\alpha}_{n p}\right)=0 .
$$

Similarly, the two eqs. (4.20) are satisfied only if $d_{n p}=0$ and, hence,

$$
\bar{\alpha}_{n p} J_{\bar{\nu}_{n}+1}\left(\bar{\alpha}_{n p}\right)-\left(2+\bar{\nu}_{n}\right) J_{\bar{\nu}_{n}}\left(\bar{\alpha}_{n p}\right)=0 .
$$

In either case, no solution exists for $n=0$, and, thus, the first nonzero components of the adjoint scalar fields would be $A_{4}^{(0,1)}\left(x_{\mu}\right)$ and $A_{5}^{(0,1)}\left(x_{\mu}\right)$.

The quantized masses that satisfy the equations. (4.26), (4.27) and (4.28) are shown in figures 3 and 4 . 

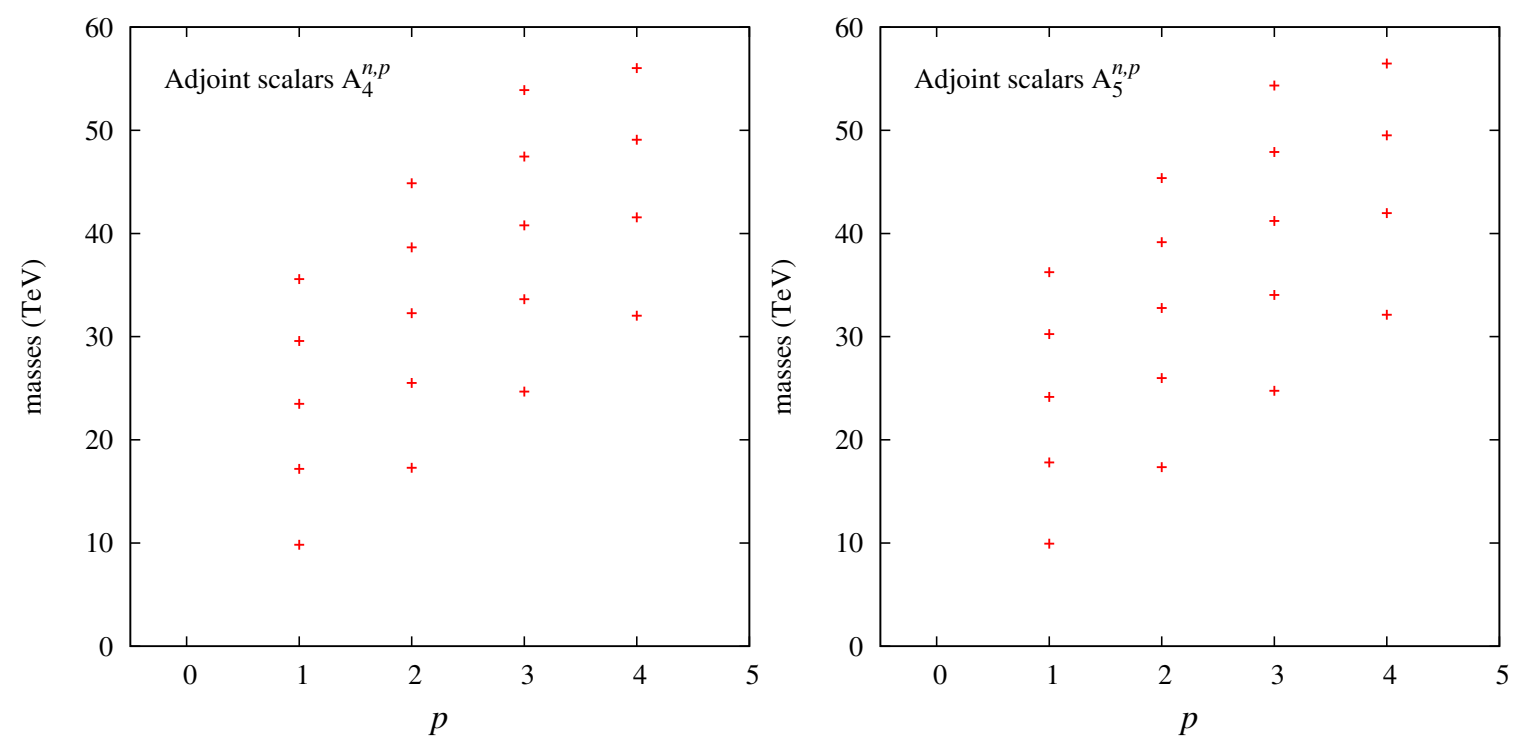

Figure 4. As in the left panel of figure 3, but for the adjoint scalars $\tilde{A}_{4}^{(n, p)}$ (left panel) and $\tilde{A}_{5}^{(n, p)}$ (right panel) instead.

\subsubsection{Large $k$ and small $c$}

Denoting $\tau=\tanh \left(k x_{5}\right)$, we may re-express the wavefunctions $\chi_{p}\left(x_{5}\right)$ as

$$
\chi_{p}(\tau)=\frac{1}{N_{p}}\left(1-\tau^{2}\right)^{\frac{3}{4}}\left[\cot \theta_{p} P_{\nu_{p}}^{u / 2}(\tau)+Q_{\nu_{p}}^{u / 2}(\tau)\right] .
$$

For $x_{5}=\pi$, we are very close to $\tau=1$. Defining $f(\tau) \equiv d \chi_{p} / d \tau$, we then have

$$
\left.f(\tau)\right|_{\tau=1-\epsilon}=\frac{1}{2 \sqrt{2 \pi}}\left(2 \nu_{p}-1\right)\left(3+2 \nu_{p}\right) \cot \theta_{p}+\mathcal{O}(\epsilon),
$$

which automatically vanishes for the zero mode as the corresponding $\nu_{p}=1 / 2$. For the higher modes to satisfy the Dirichlet boundary condition, we require $\cot \theta_{p}=0$, or in other words $\nu_{p}=2 p-1 / 2$, where $p \in \mathbb{Z}^{+}$.

Since we are now in the very small $c$ regime, the warping in the $x_{4}$ direction is virtually nonexistent, and the modes with $n \neq 0$ will have a mass $\sim n R_{y}^{-1}$ and, hence, will decouple from the theory. This is exactly analogous to the case for the corresponding graviton modes [32].

The boundary conditions for the adjoint scalars simplifies to

$$
Q_{\tilde{\nu} p}^{\tilde{v} / 2}(0) P_{\tilde{\nu}_{p}}^{\tilde{v} / 2}(\tanh k \pi)=0
$$

and

$$
Q_{\bar{\nu}_{p}}^{\bar{v} / 2}(0) P_{\bar{\nu}_{p}}^{\bar{v} / 2}(\tanh k \pi)=0,
$$

and, once again, no mode exists for $\bar{m}_{p}=0$ and $\tilde{m}_{p}=0$. Since the $n \neq 0$ modes decouple from low energy physics, the first non vanishing modes are $A_{4,5}^{(0,1)}\left(x_{\mu}\right)$. 


\subsubsection{Bulk masses}

At this stage, let us reexamine the consequences of a nonzero bulk mass term $M$ (irrespective of whether it has been occasioned by an explicit or spontaneous breaking of the symmetry). The mass parameter $M$ makes its presence felt in the context of the fourdimensional masses $m_{n p}$ primarily through the quantities $u$ and $\bar{v}$ (see eqs. (4.12), (4.14) $\&$ (4.16)). For the large $k$ case, even if $M$ is of the order of the natural cutoff, the quantities $u, v$ do not differ substantially from their $M=0$ limit values of 3 and 1 respectively. Naively, this would lead one to presume that the existence of even a seemingly large $M$ would not alter the low-lying part of the KK-tower spectrum to any significant degree. However, as has been shown in [43], this argument is fraught with danger, and much the same follows here. As can be appreciated easily, with $M$ being the bulk mass term, the mass of any given mode $m_{n p}>M$. Indeed, in the absence of warping, we would expect $m_{00}=M$ and $m_{n p}^{2}=M^{2}+n^{2} / R_{y}^{2}+p^{2} / r_{z}^{2}$. With $M^{-1}$ being of the same order as the compactification radii, the mass of the first excitation, in the absence of warping, would be of similar order as that of the zero mode. Thus, if warping is supposed to bring down the mass of the zero mode, as perceived in four-dimensions, from $M$ to $M_{Z}$ ( $Z$-boson mass), it would also, typically, bring down the first excitation to well below a $\mathrm{TeV}$, resulting in severe phenomenological contradictions. In other words, a bulk $M$, if present, would need to be much smaller than the compactification scale, thereby bringing back the hierarchy problem in a new guise. A similar argument holds for the large $c$ case too.

All of the analysis presented above carries through for a nonabelian theory as well. The additional features are the gauge boson self-interactions (a discussion of which we postpone until section 6) and ghost fields. The latter, we consider next.

\subsection{Ghosts}

As is well-known, a covariant gauge fixing condition for a nonabelian theory always gives rise to ghost fields. Accounting for the spontaneous symmetry breaking, the gauge-fixing term can be written as

$$
\begin{aligned}
\mathcal{L}_{g f} & =\frac{\sqrt{-g}}{2 \zeta} G^{a} G^{a} \\
G^{a}(A) & =\left(\frac{\eta^{\mu \nu}}{a^{2} b^{2}} \partial_{\mu} A_{\nu}^{a}+\left\{\frac{\partial_{4}\left(a^{2} b A_{4}^{a}\right)}{R_{y}^{2} a^{2} b^{3}}+\frac{\partial_{5}\left(a^{2} b^{3} A_{5}^{a}\right)}{r_{z}^{2} a^{2} b^{3}}\right\}\right)-\zeta g_{\mathrm{YM}} T_{i j}^{a} \phi_{0 i} \chi_{j},
\end{aligned}
$$

with $T_{i j}^{a}$ being the Yang Mills group generators in the representation of the scalar fields $\phi_{i}$. The latter are decomposed into the vacuum values $\phi_{0 i}$ and the perturbations $\chi_{i}$ around it $\left(\phi_{i}=\phi_{0 i}+\chi_{i}\right)$. Evidently, the gauge boson mass matrix is given by

$$
\left(M^{2}\right)^{a b}=g_{\mathrm{YM}}^{2} T_{i j}^{a} T_{k j}^{b} \phi_{0 i} \phi_{0 k}
$$

Note that the scalars $\phi_{i}$ need not be bulk fields, but may be confined to branes (as we shall argue for in section 5), with the appropriate delta-functions being included accordingly. 
Under a gauge transformation,

$$
\begin{aligned}
A_{M}^{a} \rightarrow A_{M}^{a \lambda} & =A_{M}^{a}+\frac{1}{g_{\mathrm{YM}}} \partial_{M} \theta^{a}+f^{a b c} A_{M}^{b} \lambda^{c} \\
\phi_{i} \rightarrow \phi_{i}+\delta \phi_{i} & =\phi_{i}-\lambda^{a} T_{i j}^{a} \phi_{j}
\end{aligned}
$$

where $f^{a b c}$ are the structure constants. Using the notations of ref. [51], the corresponding Faddev-Popov (FP) determinant is

$$
\begin{aligned}
& \operatorname{det}\left(\frac{\delta G^{a}\left(A^{\theta}\right)}{\delta \lambda^{b}}\right)= \\
& \quad \operatorname{det}\left(\frac{1}{g_{\mathrm{YM}}}\left[\frac{\eta^{\mu \nu}}{a^{2} b^{2}} \partial_{\mu} \mathcal{D}_{\nu}^{a b}+\frac{1}{a^{2} b^{3}}\left\{\partial_{4}\left(a^{2} b \mathcal{D}_{4}^{a b}\right)+\partial_{5}\left(a^{2} b^{3} \mathcal{D}_{5}^{a b}\right)\right\}-\left(M^{2}\right)^{a b}+\mathcal{O}(\chi)\right]\right),
\end{aligned}
$$

where $\mathcal{D}_{M}^{a c}=\partial_{M} \delta^{a c}+g f^{a b c} A_{M}^{b}$. Though they are important in their own right, since a study of the higgs interactions does not constitute the main aim of this paper, we neglect the $O(\chi)$ term. Writing the determinant as a gaussian integral over an anticommuting scalar $(\theta)$ in the adjoint representation of the gauge group yields the Lagrangian for the ghost field, namely

$$
\mathcal{L}_{\text {g.f. }}=\sqrt{-g} \bar{\theta}^{a}\left(x^{M}\right)\left[\square^{a b}-\left(M^{2}\right)^{a b}\right] \theta^{b}\left(x^{M}\right) .
$$

The ghost kinetic term, then, is

$$
\mathcal{L}_{\text {gh.kin }}=R_{y} r_{z} \bar{\theta}^{a}\left[a^{2} b^{3} \eta^{\mu \nu} \partial_{\mu} \partial_{\nu}+\frac{a^{4} b^{3}}{R_{y}^{2}}\left(\partial_{4}^{2}+\frac{2 \partial_{4} a}{a} \partial_{4}\right)+\frac{a^{4} b^{5}}{r_{z}^{2}}\left(\partial_{5}^{2}+\frac{3 \partial_{5} b}{b} \partial_{5}\right)-\left(M^{2}\right)^{a b}\right] \theta^{b} .
$$

Decomposing, in anticipation, as

$$
\theta^{a}\left(x_{\mu}, x_{4}, x_{5}\right)=\frac{1}{\sqrt{R_{y} r_{z}}} \sum_{n, p} \theta^{a(n, p)}\left(x_{\mu}\right) \eta_{n, p}\left(x_{4}\right) \chi_{p}\left(x_{5}\right),
$$

it is easy to see that $\eta_{n, p}$ and and $\chi_{p}$ satisfy the same equations of motion (and orthonormality properties) as the corresponding wavefunctions for the vector modes (see eqs. (4.10) $\&(4.11))$. In other words, not only are their masses identical to those for the corresponding vector modes, the wavefunction modes are the same too. This, of course, is as expected.

\section{Higgs}

In the preceding section, we had, for the sole purpose of determining the spectrum, included an explicit mass term for the gauge boson, without ascribing any dynamic origin to it. The simplest mechanism to generate masses while preserving a gauge symmetry, of course, is to introduce a Higgs field and effect a spontaneous breaking of the symmetry. The most straightforward, and seemingly natural, way to do so would be to consider a bulk Higgs field. However, this immediately leads to a problem (also seen in the context of bulk SM fields in the RS scenario [43]) in that the consequent masses of the first excited modes of 
the gauge bosons typically turn out to be well below ${ }^{7} 1 \mathrm{TeV}$, in stark contradiction to the direct bounds from gauge boson (most typically, generic $Z^{\prime}$ ) searches. ${ }^{8}$

The alternative, then, is to localize the Higgs on one of the 4-branes, or, perhaps even to a 3-brane at one of the junctions. A junction localized Higgs would lead to a gauge field equation of motion analogous to a two dimensional Poisson equation with a point source term. With the rotational symmetry being absent, a solution of such an equation consistent with the boundary conditions, though, is a complicated one.

\subsection{3 -brane localization}

Notwithstanding the anticipated technical problems, we begin by considering a 3 -brane localized Higgs with a Lagrangian given by

$$
\begin{aligned}
\mathcal{L}_{h} & =\delta\left(x_{4}-\pi\right) \delta\left(x_{5}\right) \sqrt{-g_{4}}\left(g^{\nu \rho} D_{\nu} \Phi\left(x^{\mu}\right)^{\dagger} D_{\rho} \Phi\left(x^{\mu}\right)+V\left(\Phi\left(x^{\mu}\right)\right)\right), \\
V(\Phi) & =\lambda\left(\Phi^{\dagger} \Phi-\tilde{v}^{2}\right)^{2} \\
D_{\mu} & =\partial_{\mu}-i g_{\mathrm{YM}} A_{\mu}\left(x_{\nu}, x_{4}, x_{5}\right) .
\end{aligned}
$$

Rewriting $\Phi\left(x^{\mu}\right)=\left[\tilde{v}+h\left(x^{\mu}\right)\right] /[a(\pi) b(0)]$ allows us to canonically quantize the Higgs field, with the Higgs mass being given by

$$
m_{h}=\sqrt{\lambda} \tilde{v} \frac{e^{-c \pi}}{\cosh (k \pi)} \equiv \sqrt{\lambda} v .
$$

Instead of solving the consequent equations of motion for the gauge fields with the delta function sources included, we consider the latter to be localized perturbations to the system with the symmetry intact. This is a valid approximation as the effect of the spontaneous breaking of the gauge symmetry is parametrized by $v$; since it is suppressed down to the electroweak scale, its contribution to the KK-gauge boson masses would be small compared to those due to compactification (even after the warping). To the first order, then, the mass spectra that we computed in section 4.1 do not get disturbed in the bulk, but for a small correction $^{9}$ due to symmetry breaking in the brane. Similarly, the wavefunction profile would change only close to the brane. With the new contribution to the gauge boson mass term being

$$
\int d x_{4} d x_{5} g_{\mathrm{YM}}^{2} \sqrt{-g} g^{\mu \nu} \delta\left(x_{4}-\pi\right) \delta\left(x_{5}\right) \Phi^{\dagger} \Phi A_{\mu} A_{\nu}
$$

it could be expressed in terms of the component fields (see eq. (4.9)) as

$$
m_{s . b .}^{2}\left(n_{1}, p_{1} ; n_{2}, p_{2}\right)=\frac{g_{\mathrm{YM}}^{2} v^{2}}{R_{y} r_{z}} \eta_{n_{1}, p_{1}}(\pi) \eta_{n_{2}, p_{2}}(\pi) \chi_{p_{1}}(0) \chi_{p_{2}}(0) .
$$

\footnotetext{
${ }^{7}$ The argument is exactly the same as the one in the preceding section arguing against a naturally large bulk mass term (howsoever generated) for the gauge bosons.

${ }^{8}$ Some of these constraints on bulk Higgs can be evaded, though, e.g. if one considers soft-wall scenarios [52-55]. However, most such constructions, typically, need additional inputs, whether it be in the form of gauge-Higgs unification or the localization of the Higgs close to the singularity, and, for certain models, even additional dynamics to generate and stabilize the soft-wall setup itself.

${ }^{9}$ This, of course, pertains only to the KK-excitations. For the zero-modes, this would be the only mass term.
} 
It comes as no surprise that this term mixes the KK modes, for this is a generic feature of brane localized Higgs fields. To the zeroth order, the gauge wavefunctions remain unchanged and have no discontinuities on the branes. Of course, to obtain the physical gauge boson states and their masses, one would need to diagonalize the mass matrix including the terms of eq. (5.3). While a closed form solution is not obvious, inspiration may be taken from the see-saw mechanism; although the hierarchy between the compactification and the Higgs contributions is not as large as in the neutrino sector, it is still sufficiently large for such approximate solutions to be valid. And while the absolute contribution of the Higgs grows for the higher- $p$ modes, primarily because $\chi_{p}$ are localized near $x_{5}=0$, this growth is relatively slow and consequently the ratio $m_{\text {s.b. }} / m_{n p}$ becomes smaller. Thus, neglecting the Higgs contribution for the higher modes is progressively a better approximation, and obtaining good estimates of the gauge boson masses and eigenstates is a relatively straightforward task.

Going beyond the first order approximation renders the algebra to be rather cumbersome, without providing us any real insight. To gain the latter, we would need to take recourse to some approximation. One such could be a smearing of the delta-function localized Higgs field.

\subsection{4-brane localization}

Choosing to work in the regime wherein the warping in the $x_{5}$-direction is small, we consider a configuration such that the factor $\delta\left(x_{5}\right)$ in eq. (5.1) is smeared onto a nonsingular but localized function. This, of course, is equivalent to considering the Higgs-field to be localized onto the 4-brane at $x_{4}=\pi$ (rather than on the 3 -brane at the junction), with a further concentration of its wavefunction close to $x_{5}=0$. The relevant part of the symmetry breaking Lagrangian can now be parametrized as

$$
\mathcal{L}_{m}=\sqrt{-g_{5}} \frac{\widetilde{M}^{2}\left(x_{5}\right)}{2} g^{\mu \nu} A_{\mu} A_{\nu} \delta(y-\pi),
$$

with $\widetilde{M}\left(x_{5}\right)$ encapsulating the $x_{5}$-dependent profile of the Higgs vacuum expectation value. Assuming, for the moment, that $\widetilde{M}=m / \sqrt{b\left(x_{5}\right)}$ where $m$ is a constant (we shall comment later on the origin of such a profile), the equations of motion for the gauge boson modes are altered from those in eq. (4.11) to

$$
\begin{aligned}
m^{2} a^{2} \delta(y-\pi) & =m_{n p}^{2}-m_{p}^{2} a^{2}+\frac{1}{R_{y}^{2} \eta_{n, p}} \partial_{4}\left(a^{2} \partial_{4} \eta_{n, p}\right) \\
-m_{p}^{2} & =\frac{1}{r_{z}^{2} b \chi_{p}} \partial_{5}\left(b^{3} \partial_{5} \chi_{p}\right)
\end{aligned}
$$

Indeed, the particular form of $\widetilde{M}\left(x_{5}\right)$ was chosen so as to render the equation of motion separable and, hence, easily solvable. The form of the second equation above is evidently the same as that in eq. (4.11) but for the bulk mass term $M$ in the latter. As for the first equation, for a given $m_{n p}$, it differs from its predecessor (see eq. (4.11)) only as far as the boundary term is concerned. Consequently, the bulk solutions are exactly the same as in 
eq. (4.12) but with $u=3$. The boundary conditions on $\chi_{p}\left(x_{5}\right)$ remain exactly the same as before (see section 4.1.2), namely

$$
\left.\chi_{p}^{\prime}\right|_{x_{5}=0}=0=\left.\chi_{p}^{\prime}\right|_{x_{5}=\pi},
$$

and, thus, the $m_{p^{-}}$-spectrum is unchanged. To obtain the full spectrum, we must consider the boundary conditions on $\eta_{n, p}$, and one of these now changes to accommodate the brane localized term, to wit,

$$
\left.\eta_{n, p}^{\prime}\right|_{x_{4}=0}=0, \quad \text { and }\left.\quad \eta_{n, p}^{\prime}\right|_{x_{4}=\pi}=m^{2} R_{y}^{2} \eta_{n, p}(\pi) .
$$

Since $m_{p=0}=0$, we have, for the modes $\eta_{n 0}$,

$J_{0}\left(e^{-c \pi} \alpha_{n 0}\right)\left[2 c \alpha_{n 0} Y_{0}\left(\alpha_{n 0}\right)+R_{y}^{2} m^{2} Y_{1}\left(\alpha_{n 0}\right)\right]=Y_{0}\left(e^{-c \pi} \alpha_{n 0}\right)\left[2 c \alpha_{n 0} J_{0}\left(\alpha_{n 0}\right)+R_{y}^{2} m^{2} J_{1}\left(\alpha_{n 0}\right)\right]$, where, as before, $\alpha_{n 0} \equiv m_{n 0} R_{y} e^{c \pi} / c$. Since the lightest mass mode is to be identified with the $W / Z$ bosons, we have $\alpha_{00} \ll 1$ (as $c \sim 10$ ). Expanding the Bessel functions, we obtain

$$
m_{00}^{2} \approx \frac{1}{2 \pi} m^{2} e^{-2 c \pi}
$$

Clearly, for the $W$ boson, $m^{2}=g_{2}^{2} v^{2}$, whereas for the $Z$ boson, $m^{2}=\left(g_{2}^{2}+g_{1}^{2}\right) v^{2}$, with $g_{2,1}$ being the weak and hypercharge coupling constants respectively.

In essence, the new boundary condition on $\eta_{n, p}$ manifests itself in a change in the gauge boson spectrum through

$$
\alpha_{n p} J_{\nu_{p}-1 / 2}\left(\alpha_{n p}\right)+\left(\frac{1}{2}+\frac{R_{y}^{2} m^{2}}{2 c}-\nu_{p}\right) J_{\nu_{p}+1 / 2}\left(\alpha_{n p}\right)=0,
$$

as distinct from eq. (4.26). With there being no singularities in the $x_{5}$-direction, all the $\chi_{p}$ modes remain unchanged (in particular, $\chi_{0}$ is flat), and all effects of the brane localized mass term for the gauge bosons manifest themselves in altering the quantized values of $\alpha_{n p}$, and, hence, in the form of $\eta_{n, p}$. Quite in parallel to the RS case [56], the change in the wavefunction for the zero modes $\left(W^{ \pm(0,0)}, Z^{(0,0)}\right)$ is concentrated close to the brane and, in magnitude, restricted to $\mathcal{O}\left(M_{W}^{2}\right)$. Away from the brane, even this relative change falls off exponentially.

As can be gleaned from the discussions in this section, a generic brane localized Higgs profile would lead to equations for the gauge bosons that do not admit simple closed form solutions. While the particular choice of $\langle\phi\rangle=v / \sqrt{b\left(x_{5}\right)}$ may seem an ad hoc one, we end this section delineating a mechanism to achieve this. The effective potential for a scalar field $\phi$ localized on the 4-brane at $x_{4}=\pi$ could be written as

$$
V_{\mathrm{eff}}=-r_{z} a^{4}(\pi) b^{4}\left[\frac{1}{2 r_{z}^{2}} \partial_{5} \phi \partial_{5} \phi+V(\phi)\right]
$$

where $V(\phi)$ is the potential term appearing in the (flat space) Lagrangian. To find what potential would give rise to the required form for $\langle\phi\rangle$, we may treat $V_{\text {eff }}$ as the Lagrangian for a one-dimensional particle. Varying with respect to $\phi$, we get

$$
\frac{1}{r_{z}^{2}} \partial_{5}\left(b^{4} \partial_{5} \phi\right)-b^{4} V^{\prime}(\phi)=0 \text {. }
$$


Demanding that the solution be of the form $\phi \propto 1 / \sqrt{b\left(x_{5}\right)}$, we have

$$
V(\phi)=\frac{k^{2}}{r_{z}^{2}}\left(\frac{5 \operatorname{sech}^{2} k \pi}{24 v^{4}} \phi^{6}-\frac{7}{8} \phi^{2}\right) .
$$

At first sight, it might seem disquieting that the form of $V(\phi)$ is fixed so uniquely. However, as previous experience has shown, such is often the case if exact closed form solutions to a complicated system (such as the gravity-scalar system under consideration here). Deviations from the form above are, of course, permissible, but only at the cost of increasing complexity of the solution (or numerical approximations). We desist from exploring such possibilities as these do not make qualitative differences to the main features of interest.

\section{Interactions}

\subsection{Gauge-fermion}

The relevant piece of the Dirac Lagrangian for the positive chirality six-dimensional field is given by

$$
\mathcal{L} \ni i g_{\mathrm{YM}} \sqrt{-g} \bar{\Psi}_{+} E_{a}^{\mu} \Gamma^{a} \Psi_{+} A_{\mu}
$$

where the group representation has been suppressed. (A similar account holds for the negative chirality field as well.) Writing the term above in its component form, we have

$$
\mathcal{L} \ni \sum_{\left\{n_{i}, p_{i}\right\}} g_{\left\{n_{i}, p_{i}\right\}}^{V, f} \bar{\psi}_{l / r}^{n_{1}, p_{1}} \gamma^{\mu} \psi_{l / r}^{n_{2}, p_{2}} A_{\mu}^{n_{3}, p_{3}},
$$

with the four dimensional charges being given by

$$
g_{\left\{n_{i}, p_{i}\right\}}^{V, f}=\frac{g_{\mathrm{YM}}}{\sqrt{R_{y} r_{z}}} \int_{0}^{\pi} d x_{4} \int_{-\pi}^{\pi} d x_{5} a^{3} b^{4} \mathcal{F}_{l / r}^{n_{1}, p_{1}}\left(x_{4}, x_{5}\right) \mathcal{F}_{l / r}^{n_{2}, p_{2}}\left(x_{4}, x_{5}\right) \eta_{n_{3}, p_{3}}\left(x_{4}\right) \chi_{p_{3}}\left(x_{5}\right) .
$$

It is evident that, for the gauge boson zero mode, we have

$$
g_{\left\{n_{i}, p_{i}\right\}}^{V, f} \rightarrow \frac{g_{\mathrm{YM}}}{\sqrt{R_{y} r_{z}}} \sqrt{\frac{k}{2 \pi \tanh (k \pi)}} \delta_{n_{1}, n_{2}} \delta_{p_{1}, p_{2}} .
$$

This universal coupling of the zero mode is, of course, mandated by gauge invariance.

For the adjoint scalar $A_{4}$, we start from

$$
\mathcal{L} \ni i g_{\mathrm{YM}} \sqrt{-g} \bar{\Psi}_{+} E_{a}^{4} \Gamma^{a} \Psi_{+} A_{4} .
$$

Rewriting in terms of the four-dimensional fields, we have

$$
\mathcal{L} \ni \sum_{\left\{n_{i}, p_{i}\right\}} g_{4, f\left\{n_{i}, p_{i}\right\}} \bar{\psi}_{l / r}^{n_{1}, p_{1}} \gamma^{5} \psi_{r / l}^{n_{2}, p_{2}} \tilde{A}_{4}^{n_{3}, p_{3}},
$$

where the four dimensional coupling constants are given by

$$
g_{4, f\left\{n_{i}, p_{i}\right\}}=\frac{g_{\mathrm{YM}}}{\sqrt{R_{y} r_{z}}} \int_{0}^{\pi} d x_{4} \int_{-\pi}^{\pi} d x_{5} a^{4} b^{4} \mathcal{F}_{l}^{n_{1}, p_{1}}\left(x_{4}, x_{5}\right) \mathcal{F}_{r}^{n_{2}, p_{2}}\left(x_{4}, x_{5}\right) \eta_{n_{3}, p_{3}}^{(4)}\left(x_{4}\right) \chi_{p_{3}}^{(4)}\left(x_{5}\right) .
$$




$k=0.3, \alpha=49, w=1.82 \times 10^{-14}$
\begin{tabular}{|c|c|c|}
\hline$(n, p)$ & $m_{n p}(\mathrm{TeV})$ & $C_{n p}$ \\
\hline$(1,0)$ & 4.47 & $3.87 \times 10^{0}$ \\
\hline$(2,0)$ & 10.2 & $4.98 \times 10^{-1}$ \\
\hline$(0,1)$ & 10.1 & $7.89 \times 10^{-1}$ \\
\hline$(1,1)$ & 17.0 & $3.03 \times 10^{-1}$ \\
\hline
\end{tabular}

$k=0.56, \alpha=50.4, w=4.48 \times 10^{-14}$
\begin{tabular}{|c|c|c|}
\hline$(n, p)$ & $m_{n p}(\mathrm{TeV})$ & $C_{n p}$ \\
\hline$(1,0)$ & 8.55 & $3.77 \times 10^{0}$ \\
\hline$(2,0)$ & 19.6 & $4.93 \times 10^{-1}$ \\
\hline$(0,1)$ & 14.6 & $2.35 \times 10^{0}$ \\
\hline$(1,1)$ & 26.9 & $7.19 \times 10^{-1}$ \\
\hline
\end{tabular}

Table 1. Sample spectra for the small $k$ case for a particular bulk curvature $(\epsilon=0.0775)$ with $R_{y}$ set to satisfy the hierarchy eq. (2.7). The ratio $C_{n p}$ (coupling of vector gauge boson to massless fermion bilinear) is as defined in eq. (6.8).

Similarly, for the adjoint scalar $A_{5}$, we start from

$$
\mathcal{L} \ni i g_{\mathrm{YM}} \sqrt{-g} \bar{\Psi}_{+} E_{a}^{5} \Gamma^{a} \Psi_{+} A_{5} .
$$

Redefining (as before) $\frac{R_{y}}{r_{z}} A_{5} \rightarrow \tilde{A}_{5}$, and writing in terms of the components, we have

$$
\mathcal{L} \ni \sum_{\left\{n_{i}, p_{i}\right\}} g_{5, f\left\{n_{i}, p_{i}\right\}} \bar{\psi}_{l / r}^{n_{1}, p_{1}} \psi_{r / l}^{n_{2}, p_{2}} \tilde{A}_{5}^{n_{3}, p_{3}},
$$

where the four dimensional coupling constants are given by

$$
g_{5, f\left\{n_{i}, p_{i}\right\}}=\frac{g_{\mathrm{YM}}}{\sqrt{R_{y} r_{z}}} \int_{0}^{\pi} d x_{4} \int_{-\pi}^{\pi} d x_{5} a^{4} b^{5} \mathcal{F}_{l}^{n_{1}, p_{1}}\left(x_{4}, x_{5}\right) \mathcal{F}_{r}^{n_{2}, p_{2}}\left(x_{4}, x_{5}\right) \eta_{n_{3}, p_{3}}^{(5)}\left(x_{4}\right) \chi_{p_{3}}^{(5)}\left(x_{5}\right) .
$$

What is of particular interest, especially in the context of collider searches, is the coupling of a relatively low-lying KK gauge boson to a pair of SM fermions (in other words, the zero modes). Some examples of gauge boson spectra and their couplings to the lowest modes of the fermion current are given in tables $1 \& 2$. The measure of importance, apart from the mass of the level- $(n, p) \mathrm{KK}$ gauge boson mass, is the scaling $C_{n p}$ of its coupling with the SM fermions, viz.

$$
C_{n p} \equiv \frac{g_{\{0,0, n\},\{0,0, p\}}^{V, f}}{g_{\{0,0,0\},\{0,0,0\}}^{V, f}} .
$$

Concentrating on the small $k$ scenario (table 1), it is interesting to note the relative closeness of the excitations (beyond the first one) as compared to the RS case with bulk gauge bosons and fermions. And as in the latter case (and unlike in the flat extradimensional scenarios), the coupling of a SM fermion pair to the gauge excitations are not universal. In particular, the coupling to the first excitation is enhanced compared to that for the zero mode, while those to the higher ones are suppressed. Furthermore, the enhancement for the $(1,0)$ mode is only slightly smaller than that for the corresponding five-dimensional theory, with this effect having only a marginal dependence on the value of $k$. This is not surprising since the leading dependence of the couplings on $k$ is common 


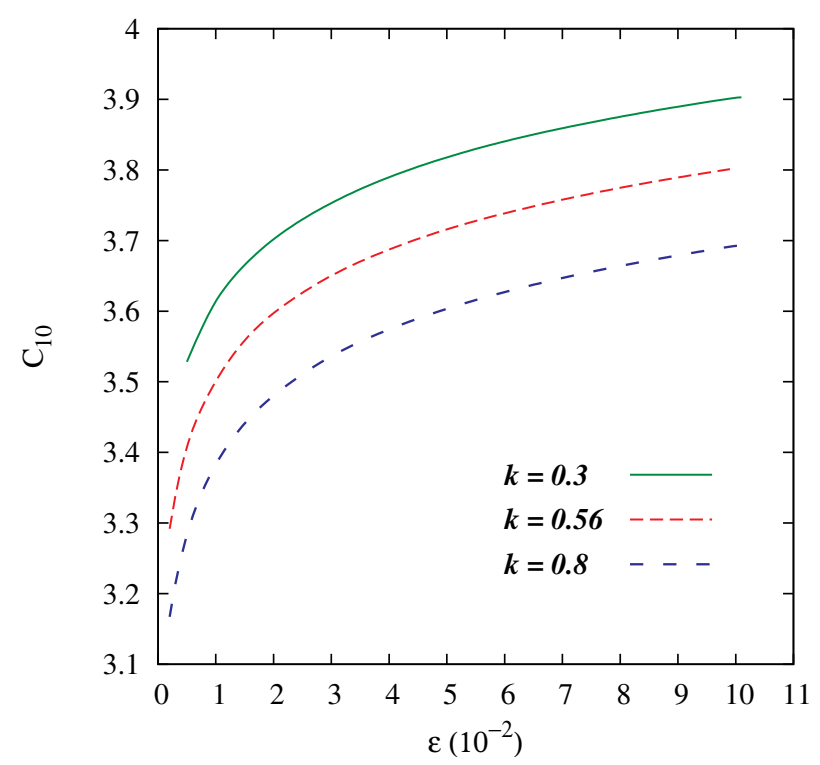

Figure 5. The matter coupling $C_{10}$ to the first vector gauge mode as a function of $\epsilon$ for a fixed $k$.

to the ground and the $(1,0)$ state. On the other hand, if the extent of warping is kept constant, the value of $c$ progressively decreases from the RS value as $k$ increases from zero. Consequently, $C_{n p}$ decreases, although only slowly. This is shown in figure 5. Although a substantial decrease in $C_{10}$ is possible (thereby making these bosons less accessible to collider searches etc.), that would require a relatively large $k$. However, as already mentioned, if $c$ and $k$ are to be of the same order, a very large hierarchy between $R_{y}$ and $r_{z}$ would be required, thereby bringing back the hierarchy problem in a different guise. In other words, the aesthetically pleasant region of the parameter space would lead to gauge bosons discoverable in the next run of the LHC.

The situation is very different in the large $k$ regime. Since the warping in the $x_{4^{-}}$ direction is very small, the $n \neq 0$ modes are all super-heavy and decouple from the TeV scale physics. In other words, just like the situation for the gravitons [32], essentially only one tower of gauge bosons (or, fermions for that matter) remains. Moreover, the couplings of the KK-tower are enhanced to nonperturbative levels. This is demonstrated, for a moderate hierarchy, in table 2. Pushing the fundamental scale $M_{6}$ even higher would, typically, result in the couplings growing even further, with the only way out of this eventuality being the introduction of a big hierarchy between the moduli. Indeed, one had seen the germ of this problem even in the graviton sector [32]. Thus, bulk fermions or gauge bosons in the large $k$ regime is ill-suited for a perturbative treatment.

\subsection{Triple gauge boson couplings}

The trilinear self interaction term can be written as

$$
\begin{gathered}
\mathcal{L} \ni g_{\mathrm{YM}} f^{a b c}\left[R_{y} r_{z} b \eta^{\mu \alpha} \eta^{\nu \rho}\left(\partial_{\mu} A_{\nu}^{a}\right) A_{\rho}^{b} A_{\alpha}^{c}+\frac{r_{z}}{R_{y}} a^{2} b \eta^{\mu \nu}\left\{\left(\partial_{\mu} A_{4}^{a}\right) A_{4}^{b} A_{\nu}^{c}+\left(\partial_{4} A_{\mu}^{a}\right) A_{4}^{b} A_{\nu}^{c}\right\}\right. \\
\left.+\frac{R_{y}}{r_{z}} a^{2} b^{3} \eta^{\mu \nu}\left\{\left(\partial_{\mu} A_{5}^{a}\right) A_{5}^{b} A_{\nu}^{c}+\left(\partial_{5} A_{\mu}^{a}\right) A_{5}^{b} A_{\nu}^{c}\right\}\right],
\end{gathered}
$$




$k=6, \alpha=16.8, \epsilon=0.001$
\begin{tabular}{|c|c|c|}
\hline$(n, p)$ & $m_{n p}(\mathrm{TeV})$ & $C_{n p}$ \\
\hline$(0,1)$ & 22.0 & $1.71 \times 10^{3}$ \\
\hline$(0,2)$ & 49.1 & $3.06 \times 10^{2}$ \\
\hline$(0,3)$ & 75.0 & $1.29 \times 10^{2}$ \\
\hline
\end{tabular}

Table 2. Sample spectrum for the large $k$ case with $R_{y}$ set to satisfy the hierarchy eq. (2.7). The ratio $C_{n p}$ is as defined in eq. (6.8).

where $f^{a b c}$ are the structure constants. The six-dimensional Ward identities ensure that the coupling $g_{\mathrm{YM}}$ equals the six-dimensional gauge-fermion coupling even after quantum corrections are included. Rewriting in terms of the four dimensional fields, we have

$$
\begin{aligned}
\mathcal{L} \ni f^{a b c} \sum_{\left\{n_{i}, p_{i}\right\}}[ & g_{\left\{n_{i}, p_{i}\right\}}^{(3 v)} \eta^{\mu \alpha} \eta^{\nu \rho}\left(\partial_{\mu} A_{\nu}^{a\left(n_{1}, p_{1}\right)}\right) A_{\rho}^{b\left(n_{2}, p_{2}\right)} A_{\alpha}^{c\left(n_{3}, p_{3}\right)} \\
& +g_{\left\{n_{i}, p_{i}\right\}}^{(v v 4)} \eta^{\mu \nu} A_{\mu}^{a\left(n_{1}, p_{1}\right)} A_{\nu}^{b\left(n_{2}, p_{2}\right)} \tilde{A}_{4}^{c\left(n_{3}, p_{3}\right)} \\
& +g_{\left\{n_{i}, p_{i}\right\}}^{(4 v)} \eta^{\mu \nu}\left(\partial_{\mu} \tilde{A}_{4}^{a\left(n_{1}, p_{1}\right)}\right) \tilde{A}_{4}^{b\left(n_{2}, p_{2}\right)} A_{\nu}^{c\left(n_{3}, p_{3}\right)} \\
& +g_{\left\{n_{i}, p_{i}\right\}}^{(v v)} \eta^{\mu \nu} A_{\mu}^{a\left(n_{1}, p_{1}\right)} A_{\nu}^{b\left(n_{2}, p_{2}\right)} \tilde{A}_{5}^{c\left(n_{3}, p_{3}\right)} \\
& \left.+g_{\left\{n_{i}, p_{i}\right\}}^{(55 v)} \eta^{\mu \nu}\left(\partial_{\mu} \tilde{A}_{5}^{a\left(n_{1}, p_{1}\right)}\right) \tilde{A}_{5}^{b\left(n_{2}, p_{2}\right)} A_{\nu}^{c\left(n_{3}, p_{3}\right)}\right],
\end{aligned}
$$

where the coupling constants are defined through

$$
\begin{aligned}
& g_{\left\{n_{i}, p_{i}\right\}}^{(3 v)}=\frac{g_{\mathrm{YM}}}{\sqrt{R_{y} r_{z}}} \int_{0}^{\pi} d x_{4} \eta_{n_{1}, p_{1}} \eta_{n_{2}, p_{2}} \eta_{n_{3}, p_{3}} \int_{-\pi}^{\pi} d x_{5} b \chi_{p_{1}} \chi_{p_{2}} \chi_{p_{3}}, \\
& g_{\left\{n_{i}, p_{i}\right\}}^{(v v 4)}=\frac{g_{\mathrm{YM}}}{R_{y}^{2}} \sqrt{\frac{R_{y}}{r_{z}}} \int_{0}^{\pi} d x_{4} a^{2} \eta_{n_{1}, p_{1}} \eta_{n_{2}, p_{2}} \eta_{n_{3}, p_{3}}^{(4)} \int_{-\pi}^{\pi} d x_{5} b\left(\partial_{4} \chi_{p_{1}}\right) \chi_{p_{2}} \chi_{p_{3}}^{(4)}, \\
& g_{\left\{n_{i}, p_{i}\right\}}^{(44 v)}=\frac{g_{\mathrm{YM}}}{\sqrt{R_{y} r_{z}}} \int_{0}^{\pi} d x_{4} a^{2} \eta_{n_{1}, p_{1}}^{(4)} \eta_{n_{2}, p_{2}}^{(4)} \eta_{n_{3}, p_{3}} \int_{-\pi}^{\pi} d x_{5} b \chi_{p_{1}}^{(4)} \chi_{p_{2}}^{(4)} \chi_{p_{3}}, \\
& g_{\left\{n_{i}, p_{i}\right\}}^{(v v 5)}=\frac{g_{\mathrm{YM}}}{r_{z}^{2}} \sqrt{\frac{r_{z}}{R_{y}}} \int_{0}^{\pi} d x_{4} a^{2} \eta_{n_{1}, p_{1}} \eta_{n_{2}, p_{2}} \eta_{n_{3}, p_{3}}^{(5)} \int_{-\pi}^{\pi} d x_{5} b^{3}\left(\partial_{5} \chi_{p_{1}}\right) \chi_{p_{2}} \chi_{p_{3}}^{(5)}, \\
& g_{\left\{n_{i}, p_{i}\right\}}^{(55 v)}=\frac{g_{\mathrm{YM}}}{\sqrt{R_{y} r_{z}}} \int_{0}^{\pi} d x_{4} a^{2} \eta_{n_{1}, p_{1}}^{(5)} \eta_{n_{2}, p_{2}}^{(5)} \eta_{n_{3}, p_{3}} \int_{-\pi}^{\pi} d x_{5} b^{3} \chi_{p_{1}}^{(5)} \chi_{p_{2}}^{(5)} \chi_{p_{3}} \ldots
\end{aligned}
$$

For the three vector vertex, clearly if one of them is a zero-mode, the other two must be identical. Similarly, the vector zero-mode couples only to a pair of identical scalars. Finally, for either case, the coupling is the same as that in eq. (6.3). All of the above are, of course, consequences of gauge invariance. Finally, although there exists $\tilde{A}_{4}-\tilde{A}_{5}$ mixing term, it, as discussed earlier, is rather subdominant, and we omit it here. 


\subsection{Quartic gauge interaction}

The corresponding term in the Lagrangian is

$$
\mathcal{L} \ni g_{\mathrm{YM}}^{2} f^{a b c} f^{c e d} R_{y} r_{z} \eta^{\mu \rho} A_{\mu}^{a} A_{\rho}^{e}\left(b \eta^{\nu \alpha} A_{\nu}^{b} A_{\alpha}^{d}+\frac{a^{2} b}{R_{y}^{2}} A_{4}^{b} A_{4}^{d}+\frac{a^{2} b^{3}}{r_{z}^{2}} A_{5}^{b} A_{5}^{d}\right) .
$$

Once again, reexpressing in terms of four dimensional fields, we have

$$
\begin{aligned}
& \mathcal{L} \ni f^{a b c} f^{c e d} \eta^{\mu \rho} \sum_{\left\{n_{i}, p_{i}\right\}} A_{\mu}^{a\left(n_{1}, p_{1}\right)} A_{\rho}^{e\left(n_{3}, p_{3}\right)} {\left[\mathcal{G}_{\left\{n_{i}, p_{i}\right\}}^{(4 v)} \eta^{\nu \alpha} A_{\nu}^{b\left(n_{2}, p_{2}\right)} A_{\alpha}^{d\left(n_{4}, p_{4}\right)}\right.} \\
&\left.+\mathcal{G}_{\left\{n_{i}, p_{i}\right\}}^{4(2 v, 2 s)} \tilde{A}_{4}^{b\left(n_{2}, p_{2}\right)} \tilde{A}_{4}^{d\left(n_{4}, p_{4}\right)}+\mathcal{G}_{\left\{n_{i}, p_{i}\right\}}^{5(2 v, 2 s)} \tilde{A}_{5}^{b\left(n_{2}, p_{2}\right)} \tilde{A}_{5}^{d\left(n_{4}, p_{4}\right)}\right]
\end{aligned}
$$

with the coupling constants being defined through

$$
\begin{aligned}
\mathcal{G}_{\left\{n_{i}, p_{i}\right\}}^{(4 v)} & =\frac{g_{\mathrm{YM}}^{2}}{R_{y} r_{z}} \int_{0}^{\pi} d x_{4} \eta_{n_{1}, p_{1}} \eta_{n_{2}, p_{2}} \eta_{n_{3}, p_{3}} \eta_{n_{4}, p_{4}} \int_{-\pi}^{\pi} d x_{5} b \chi_{p_{1}} \chi_{p_{2}} \chi_{p_{3}} \chi_{p_{4}}, \\
\mathcal{G}_{\left\{n_{i}, p_{i}\right\}}^{4(2 v, 2 s)} & =\frac{g_{\mathrm{YM}}^{2}}{R_{y} r_{z}} \int_{0}^{\pi} d x_{4} a^{2} \eta_{n_{1}, p_{1}} \eta_{n_{3}, p_{3}} \eta_{n_{2}, p_{2}}^{(4)} \eta_{n_{4}, p_{4}}^{(4)} \int_{-\pi}^{\pi} d x_{5} b \chi_{p_{1}} \chi_{p_{3}} \chi_{p_{2}}^{(4)} \chi_{p_{4}}^{(4)}, \\
\mathcal{G}_{\left\{n_{i}, p_{i}\right\}}^{5(2 v, 2 s)} & =\frac{g_{\mathrm{YM}}^{2}}{R_{y} r_{z}} \int_{0}^{\pi} d x_{4} a^{2} \eta_{n_{1}, p_{1}} \eta_{n_{3}, p_{3}} \eta_{n_{2}, p_{2}}^{(5)} \eta_{n_{4}, p_{4}}^{(5)} \int_{-\pi}^{\pi} d x_{5} b^{3} \chi_{p_{1}} \chi_{p_{3}} \chi_{p_{2}}^{(5)} \chi_{p_{4}}^{(5)} .
\end{aligned}
$$

Again, for the zero mode vectors, the Ward identity is satisfied.

\subsection{Ghost vertices}

The relevant piece in the ghost Lagrangian is

$$
\begin{gathered}
\mathcal{L}_{g h} \ni g_{\mathrm{YM}} f^{a b c} R_{y} r_{z} \bar{\theta}^{a}\left(a^{2} b^{3} \eta^{\mu \nu} \partial_{\mu} A_{\nu}^{b}+\frac{1}{R_{y}^{2}} a^{3} b^{3}\left(\partial_{4} a\right) A_{4}^{b}+\frac{1}{R_{y}^{2}} a^{4} b^{3} \partial_{4} A_{4}^{b}\right. \\
\left.+\frac{1}{r_{z}^{2}} a^{4} b^{5} \partial_{5} A_{5}^{b}+\frac{3}{r_{z}^{2}} a^{4} b^{4}\left(\partial_{5} b\right) A_{5}^{b}\right) \theta^{c} .
\end{gathered}
$$

yielding in terms of the four dimensional fields

$$
\begin{aligned}
\mathcal{L}_{g h} \ni & f^{a b c} \sum_{\left\{n_{i}, p_{i}\right\}} \bar{\theta}^{a\left(n_{1}, p_{1}\right)} \theta^{c\left(n_{2}, p_{2}\right)}\left[g_{\left\{n_{i}, p_{i}\right\}}^{(1)} \eta^{\mu \nu} \partial_{\mu} A_{\nu}^{b\left(n_{3}, p_{3}\right)}+g_{\left\{n_{i}, p_{i}\right\}}^{(2)} \tilde{A}_{4}^{b\left(n_{3}, p_{3}\right)}\right. \\
& \left.+g_{\left\{n_{i}, p_{i}\right\}}^{(3)} \tilde{A}_{4}^{b\left(n_{3}, p_{3}\right)}+g_{\left\{n_{i}, p_{i}\right\}}^{(4)} \tilde{A}_{5}^{b\left(n_{3}, p_{3}\right)}+g_{\left\{n_{i}, p_{i}\right\}}^{(5)} \tilde{A}_{5}^{b\left(n_{3}, p_{3}\right)}\right]
\end{aligned}
$$

where the coupling constants are defined as

$$
\begin{aligned}
& g_{\left\{n_{i}, p_{i}\right\}}^{(1)}=\frac{g_{\mathrm{YM}}}{\sqrt{R_{y} r_{z}}} \int_{0}^{\pi} d x_{4} a^{2} \eta_{n_{1}, p_{1}} \eta_{n_{2}, p_{2}} \eta_{n_{3}, p_{3}} \int_{-\pi}^{\pi} d x_{5} b^{3} \chi_{p_{1}} \chi_{p_{2}} \chi_{p_{3}}, \\
& g_{\left\{n_{i}, p_{i}\right\}}^{(2)}=\frac{g_{\mathrm{YM}}}{R_{y} \sqrt{R_{y} r_{z}}} \int_{0}^{\pi} d x_{4} a^{3} a^{\prime} \eta_{n_{1}, p_{1}} \eta_{n_{2}, p_{2}} \eta_{n_{3}, p_{3}}^{(4)} \int_{-\pi}^{\pi} d x_{5} b^{3} \chi_{p_{1}} \chi_{p_{2}} \chi_{p_{3}}^{(4)}, \\
& g_{\left\{n_{i}, p_{i}\right\}}^{(3)}=\frac{g_{\mathrm{YM}}}{R_{y} \sqrt{R_{y} r_{z}}} \int_{0}^{\pi} d x_{4} a^{4} \eta_{n_{1}, p_{1}} \eta_{n_{2}, p_{2}} \partial_{4} \eta_{n_{3}, p_{3}}^{(4)} \int_{-\pi}^{\pi} d x_{5} b^{3} \chi_{p_{1}} \chi_{p_{2}} \chi_{p_{3}}^{(4)}, \\
& g_{\left\{n_{i}, p_{i}\right\}}^{(4)}=\frac{g_{\mathrm{YM}}}{r_{z} \sqrt{R_{y} r_{z}}} \int_{0}^{\pi} d x_{4} a^{4} \eta_{n_{1}, p_{1}} \eta_{n_{2}, p_{2}} \eta_{n_{3}, p_{3}}^{(5)} \int_{-\pi}^{\pi} d x_{5} b^{5} \chi_{p_{1}} \chi_{p_{2}} \partial_{5} \chi_{p_{3}}^{(5)}, \\
& g_{\left\{n_{i}, p_{i}\right\}}^{(5)}=\frac{g_{\mathrm{YM}}}{r_{z} \sqrt{R_{y} r_{z}}} \int_{0}^{\pi} d x_{4} a^{4} \eta_{n_{1}, p_{1}} \eta_{n_{2}, p_{2}} \eta_{n_{3}, p_{3}}^{(5)} \int_{-\pi}^{\pi} d x_{5} b^{4} \dot{b} \chi_{p_{1}} \chi_{p_{2}} \chi_{p_{3}}^{(5)} .
\end{aligned}
$$

Once, again, $g_{\overrightarrow{0}, \overrightarrow{0}}^{(1)}=g_{\overrightarrow{0}, \overrightarrow{0}}^{(3 v)}$, as is mandated by gauge invariance. 


\section{Summary}

While the negative results for graviton resonance searches by the ATLAS [9] and CMS [10] collaborations have caused a bit of tension for the Randall-Sundrum scenario, a sixdimensional analogue with a nested double warping [31] has been seen to be very consistent with the experimental results [32]. Such scenarios are of interest in their own right as they could, for example, constitute an intermediate step in the compactification down from a theory in higher dimensions. Moreover, the fundamental scale in such theories are naturally lower than the Planck-scale, and this could play a significant role in the context of gauge unification. But, most interestingly, it provides a tunable parameter that smoothly takes one from a nearly-conformal theory to another that is a large departure from one, with the added feature that both the ends provide a resolution of the hierarchy problem (although this is not apparent in the interim regime).

Just as the RS model would, generically, admit operators that lead to unsuppressed flavour changing neutral currents and/or proton decay, so would the model considered in refs. [31, 32]. On a different vein, the exact cutoff scale of this theory (normally described as the scale at which the loop contributions are to be cutoff) needs to be identified too. It has been argued that, within the five-dimensional context, the addition of the Planck-brane and/or the TeV-brane allows a holographic interpretation [58-60], with the former acting as a regulator leading to an ultraviolet ${ }^{10}$ cutoff $\left(\lesssim r_{c}^{-1}\right)$ on the corresponding CFT [61-63]. It has been demonstrated that, for RS-like theories with gauge fields extended in to the warped bulk, this is indeed so [64-66]. Even though no such duality has been constructed for the case under consideration, it is quite conceivable that one such would exist. In the large $k$ case, the bulk is indeed $A d S_{6}$-like. However, for the phenomenologically more interesting case of large $c$ (small $k$ ), it is evident that the the metric is not conformally flat and, hence, a holographic interpretation would be considerably more tricky.

An alternative and obvious way to ameliorate flavour changing currents is to allow the fermions (and, hence, the gauge fields too) to propagate into the six dimensional bulk, for now the higher dimensional operators get suppressed by a factor $\Lambda_{\mathrm{UV}}^{4}$, with $\Lambda_{\mathrm{UV}}$ being the bulk cutoff of the theory, which is higher than the GUT scale. Furthermore, a sixdimensional theory allows one to make predictions about the number of chiral generations in the theory.

An immediate consequence of taking these fields into the full six-dimensional bulk is that each of the KK-towers that are so familiar in the five-dimensional context now expand into a "tower of towers", thereby enriching the phenomenology, whether it be in the context of quantum correcions to SM amplitudes or direct production at, say the next run of the LHC. In this paper, we have derived the wave profiles for these fields and computed the master formula to calculate their spectra. It is seen that, of the two branches allowed to the theory by the resolution of the hierarchy problem, the one close to a conformally flat space leads to the collapse of the 'tower of towers' (for both the gauge fields and the fermions) to a single tower each (the other excitations are too heavy and decouple from

\footnotetext{
${ }^{10}$ An analogous argument for our case would imply a cutoff $\Lambda_{U V} \simeq \min \left(R_{y}^{-1}, r_{z}^{-1}\right)$ as argued in ref. [32] from an entirely different perspective.
} 
the low-energy theory). However, the spacing between the successive members of a tower is distinctly different from that in the five-dimensional analogue, thereby distinguishing between the two scenarios. More tellingly though, the higher KK-excitations of the gauge bosons couple very strongly, thereby invalidating a perturbative treatment, and calls for a more sophisticated approach.

The other branch of the theory, which cannot be thought of as a mere perturbation of the $\mathrm{AdS}_{6}$ paradigm, retains the full tower of towers, with some of the excitations possibly being rather closely spaced. This naturally leads to very interesting phenomenology, not the least of which pertains to the just-begun run of the LHC. It also is of paramount interest in the context of electroweak precision tests, rare decays and renormalization group evolutions [67]. A particularly interesting feature is that the first KK mode for the fermions as well as the adjoint scalar is much heavier than that for the gauge boson. This is very different from the case of the five-dimensional theory and constitutes a remarkable discriminant between the theories, say at the LHC.

As for the Higgs field, just as in the five-dimensional theory, putting the Higgs field along with symmetry breaking potential into the bulk either brings back the hierarchy problem, or renders the masses of the gauge boson KK-tower unacceptably low. Thus, it is wise to localize the Higgs on to a 3-brane. This, though, has the unfortunate consequence of making the combination of the equations of motion and the boundary condition too complicated to permit an easy understanding of the dynamics. On the other hand, if we localize the Higgs onto a 4-brane (located at $x_{4}=\pi$ and $x_{5}=0$ ), and work in the large $c$ regime, we see that the Higgs vacuum expectation value does get warped down to the electroweak scale. With the consequent brane localized contribution being small, it can be treated as a perturbation, and the consequent shifts in the spectrum as well as the wavefunction profiles can be calculated. It is interesting to note that, although small, the brane localized Higgs mixes the KK states with possible phenomenological ramifications. These issues are under investigation.

\section{Acknowledgments}

MTA would like to thank UGC-CSIR, India for assistance under Senior Research Fellowship Grant Sch/SRF/AA/139/F-123/2011-12. DC acknowledges partial support from the European Union FP7 ITN INVISIBLES (Marie Curie Actions, PITN- GA-2011- 289442), and the Research and Development grant of the University of Delhi. 
Open Access. This article is distributed under the terms of the Creative Commons Attribution License (CC-BY 4.0), which permits any use, distribution and reproduction in any medium, provided the original author(s) and source are credited.

\section{References}

[1] ATLAS collaboration, Observation of a new particle in the search for the Standard Model Higgs boson with the ATLAS detector at the LHC, Phys. Lett. B 716 (2012) 1 [arXiv:1207.7214] [INSPIRE].

[2] ATLAS collaboration, Combined search for the Standard Model Higgs boson in pp collisions at $\sqrt{s}=7 \mathrm{TeV}$ with the ATLAS detector, Phys. Rev. D 86 (2012) 032003 [arXiv:1207.0319] [INSPIRE].

[3] CMS collaboration, Observation of a new boson at a mass of $125 \mathrm{GeV}$ with the CMS experiment at the LHC, Phys. Lett. B 716 (2012) 30 [arXiv:1207.7235] [INSPIRE].

[4] G. Nordström, Über die Möglichkeit, das elektromagnetische Feld und das Gravitationsfeld zu vereinigen, Phys. Zeit. 15 (1914) 504.

[5] T. Kaluza, Zum Unitätsproblem in der Physik, Sitzungsber. Preuss. Akad. Wiss. Berlin. (Math. Phys.) (1921) 966.

[6] O. Klein, Quantum Theory and Five-Dimensional Theory of Relativity. (In German and English), Z. Phys. 37 (1926) 895 [INSPIRE].

[7] L. Randall and R. Sundrum, A large mass hierarchy from a small extra dimension, Phys. Rev. Lett. 83 (1999) 3370 [hep-ph/9905221] [INSPIRE].

[8] L. Randall and R. Sundrum, An Alternative to compactification, Phys. Rev. Lett. 83 (1999) 4690 [hep-th/9906064] [inSPIRE].

[9] ATLAS collaboration, Search for Extra Dimensions in diphoton events using proton-proton collisions recorded at $\sqrt{s}=7 \mathrm{TeV}$ with the ATLAS detector at the LHC, New J. Phys. 15 (2013) 043007 [arXiv: 1210.8389] [INSPIRE].

[10] CMS collaboration, Search for massive resonances decaying into pairs of boosted bosons in semi-leptonic final states at $\sqrt{s}=8 \mathrm{TeV}$, JHEP 08 (2014) 174 [arXiv:1405.3447] [INSPIRE].

[11] S. Randjbar-Daemi and M.E. Shaposhnikov, On some new warped brane world solutions in higher dimensions, Phys. Lett. B 491 (2000) 329 [hep-th/0008087] [INSPIRE].

[12] N. Kaloper, Origami world, JHEP 05 (2004) 061 [hep-th/0403208] [INSPIRE].

[13] T. Gherghetta and A. Kehagias, Anomaly free brane worlds in seven-dimensions, Phys. Rev. Lett. 90 (2003) 101601 [hep-th/0211019] [INSPIRE].

[14] Z. Chacko and A.E. Nelson, A solution to the hierarchy problem with an infinitely large extra dimension and moduli stabilization, Phys. Rev. D 62 (2000) 085006 [hep-th/9912186] [INSPIRE].

[15] A.G. Cohen and D.B. Kaplan, Solving the hierarchy problem with noncompact extra dimensions, Phys. Lett. B 470 (1999) 52 [hep-th/9910132] [INSPIRE].

[16] R. Gregory, Nonsingular global string compactifications, Phys. Rev. Lett. 84 (2000) 2564 [hep-th/9911015] [INSPIRE]. 
[17] S.M. Carroll, S. Hellerman and M. Trodden, BPS domain wall junctions in infinitely large extra dimensions, Phys. Rev. D 62 (2000) 044049 [hep-th/9911083] [InSPIRE].

[18] N. Arkani-Hamed, L.J. Hall, D. Tucker-Smith and N. Weiner, Solving the hierarchy problem with exponentially large dimensions, Phys. Rev. D 62 (2000) 105002 [hep-ph/9912453] [INSPIRE].

[19] T. Gherghetta and M.E. Shaposhnikov, Localizing gravity on a string-like defect in six-dimensions, Phys. Rev. Lett. 85 (2000) 240 [hep-th/0004014] [InSPIRE].

[20] M. Giovannini, H. Meyer and M.E. Shaposhnikov, Warped compactification on Abelian vortex in six-dimensions, Nucl. Phys. B 619 (2001) 615 [hep-th/0104118] [INSPIRE].

[21] C.P. Burgess, J.M. Cline, N.R. Constable and H. Firouzjahi, Dynamical stability of six-dimensional warped brane worlds, JHEP 01 (2002) 014 [hep-th/0112047] [INSPIRE].

[22] M. Giovannini, Gauge field localization on Abelian vortices in six-dimensions, Phys. Rev. D 66 (2002) 044016 [hep-th/0205139] [INSPIRE].

[23] M. Giovannini, Scalar normal modes of higher dimensional gravitating kinks, Class. Quant. Grav. 20 (2003) 1063 [gr-qc/0207116] [INSPIRE].

[24] P. Kanti, R. Madden and K.A. Olive, A 6-D brane world model, Phys. Rev. D 64 (2001) 044021 [hep-th/0104177] [INSPIRE].

[25] T.G. Rizzo, Introduction to Extra Dimensions, AIP Conf. Proc. 1256 (2010) 27 [arXiv: 1003.1698] [INSPIRE].

[26] Z. Chacko, P.J. Fox, A.E. Nelson and N. Weiner, Large extra dimensions from a small extra dimension, JHEP 03 (2002) 001 [hep-ph/0106343] [INSPIRE].

[27] F. Chen, J.M. Cline and S. Kanno, Modified Friedmann Equation and Inflation in Warped Codimension-two Braneworld, Phys. Rev. D 77 (2008) 063531 [arXiv:0801.0226] [InSPIRE].

[28] K.L. McDonald, Little Randall-Sundrum Model and a Multiply Warped Spacetime, Phys. Rev. D 77 (2008) 124046 [arXiv:0804.0654] [INSPIRE].

[29] A.A. Saharian, Surface Casimir densities and induced cosmological constant in higher dimensional braneworlds, Phys. Rev. D 74 (2006) 124009 [hep-th/0608211] [InSPIRE].

[30] P. Midodashvili, Gravitational trapping of matters in $6 D$ and three fermion generations, Europhys. Lett. 66 (2004) 478 [INSPIRE].

[31] D. Choudhury and S. SenGupta, Living on the edge in a spacetime with multiple warping, Phys. Rev. D 76 (2007) 064030 [hep-th/0612246] [INSPIRE].

[32] M.T. Arun, D. Choudhury, A. Das and S. SenGupta, Graviton modes in multiply warped geometry, Phys. Lett. B 746 (2015) 266 [arXiv:1410.5591] [InSPIRE].

[33] B.A. Dobrescu and E. Poppitz, Number of fermion generations derived from anomaly cancellation, Phys. Rev. Lett. 87 (2001) 031801 [hep-ph/0102010] [INSPIRE].

[34] T. Appelquist, B.A. Dobrescu, E. Ponton and H.-U. Yee, Proton stability in six-dimensions, Phys. Rev. Lett. 87 (2001) 181802 [hep-ph/0107056] [InSPIRE].

[35] V.A. Rubakov and M.E. Shaposhnikov, Extra Space-Time Dimensions: Towards a Solution to the Cosmological Constant Problem, Phys. Lett. B 125 (1983) 139 [INSPIRE].

[36] G. Burdman, B.A. Dobrescu and E. Ponton, Resonances from two universal extra dimensions, Phys. Rev. D 74 (2006) 075008 [hep-ph/0601186] [INSPIRE]. 
[37] B.A. Dobrescu, K. Kong and R. Mahbubani, Leptons and photons at the LHC: Cascades through spinless adjoints, JHEP 07 (2007) 006 [hep-ph/0703231] [INSPIRE].

[38] A. Freitas and K. Kong, Two universal extra dimensions and spinless photons at the ILC, JHEP 02 (2008) 068 [arXiv:0711.4124] [INSPIRE].

[39] D. Choudhury, A. Datta, D.K. Ghosh and K. Ghosh, Exploring two Universal Extra Dimensions at the CERN LHC, JHEP 04 (2012) 057 [arXiv:1109.1400] [INSPIRE].

[40] B.A. Dobrescu, D. Hooper, K. Kong and R. Mahbubani, Spinless photon dark matter from two universal extra dimensions, JCAP 10 (2007) 012 [arXiv:0706.3409] [INSPIRE].

[41] G. Cacciapaglia, A. Deandrea and J. Llodra-Perez, A Dark Matter candidate from Lorentz Invariance in 6D, JHEP 03 (2010) 083 [arXiv:0907.4993] [INSPIRE].

[42] G. Cacciapaglia, A. Deandrea and J. Llodra-Perez, The Universal Real Projective Plane: LHC phenomenology at one Loop, JHEP 10 (2011) 146 [arXiv:1104.3800] [INSPIRE].

[43] S. Chang, J. Hisano, H. Nakano, N. Okada and M. Yamaguchi, Bulk standard model in the Randall-Sundrum background, Phys. Rev. D 62 (2000) 084025 [hep-ph/9912498] [INSPIRE].

[44] T.G. Rizzo and J.D. Wells, Electroweak precision measurements and collider probes of the standard model with large extra dimensions, Phys. Rev. D 61 (2000) 016007 [hep-ph/9906234] [INSPIRE].

[45] A. Das, R.S. Hundi and S. SenGupta, Bulk Higgs and Gauge fields in a multiply warped braneworld model, Phys. Rev. D 83 (2011) 116003 [arXiv:1105.1064] [INSPIRE].

[46] M. Gogberashvili and D. Singleton, Nonsingular increasing gravitational potential for the brane in 6-D, Phys. Lett. B 582 (2004) 95 [hep-th/0310048] [INSPIRE].

[47] M. Gogberashvili and D. Singleton, Brane in 6-D with increasing gravitational trapping potential, Phys. Rev. D 69 (2004) 026004 [hep-th/0305241] [InSPIRE].

[48] M. Gogberashvili, P. Midodashvili and D. Singleton, Fermion Generations from 'Apple-Shaped' Extra Dimensions, JHEP 08 (2007) 033 [arXiv:0706.0676] [InSPIRE].

[49] T. Gherghetta and A. Pomarol, Bulk fields and supersymmetry in a slice of AdS, Nucl. Phys. B 586 (2000) 141 [hep-ph/0003129] [InSPIRE].

[50] G. Burdman, B.A. Dobrescu and E. Ponton, Six-dimensional gauge theory on the chiral square, JHEP 02 (2006) 033 [hep-ph/0506334] [INSPIRE].

[51] M.E. Peskin and D.V. Schroeder, An Introduction to Quantum Field Theory, First Indian ed., Levant Books, (2005).

[52] A. Falkowski and M. Pérez-Victoria, Electroweak Breaking on a Soft Wall, JHEP 12 (2008) 107 [arXiv:0806.1737] [INSPIRE].

[53] B. Batell, T. Gherghetta and D. Sword, The Soft-Wall Standard Model, Phys. Rev. D 78 (2008) 116011 [arXiv:0808.3977] [INSPIRE].

[54] J.A. Cabrer, G. von Gersdorff and M. Quirós, Soft-Wall Stabilization, New J. Phys. 12 (2010) 075012 [arXiv:0907.5361] [INSPIRE].

[55] P.R. Archer, S.J. Huber and S. Jager, Flavour Physics in the Soft Wall Model, JHEP 12 (2011) 101 [arXiv:1108.1433] [INSPIRE].

[56] C. Csáki, J. Erlich and J. Terning, The Effective Lagrangian in the Randall-Sundrum model and electroweak physics, Phys. Rev. D 66 (2002) 064021 [hep-ph/0203034] [INSPIRE]. 
[57] R.S. Hundi and S. SenGupta, Fermion mass hierarchy in a multiple warped braneworld model, J. Phys. G 40 (2013) 075002 [arXiv:1111.1106] [InSPIRE].

[58] J.M. Maldacena, The Large- $N$ limit of superconformal field theories and supergravity, Int. J. Theor. Phys. 38 (1999) 1113 [hep-th/9711200] [INSPIRE].

[59] H. Verlinde, Holography and Compactification, invited talk at New Dimensions in Field Theory and String Theory, ITP Santa Barbara Conference, 17-20 November 1999, http://www.itp.ucsb.edu/online/susy_c99/verlinde.

[60] E. Witten, Comments by E. Witten on Sundrum $\mathcal{E}$ Giddings talk, in New Dimensions in Field Theory and String Theory, ITP Santa Barbara Conference, 17-20 November 1999, http://www.itp.ucsb.edu/online/susy_c99/discussion.

[61] N. Arkani-Hamed, M. Porrati and L. Randall, Holography and phenomenology, JHEP 08 (2001) 017 [hep-th/0012148] [INSPIRE].

[62] R. Rattazzi and A. Zaffaroni, Comments on the holographic picture of the Randall-Sundrum model, JHEP 04 (2001) 021 [hep-th/0012248] [INSPIRE].

[63] M. Pérez-Victoria, Randall-Sundrum models and the regularized AdS/CFT correspondence, JHEP 05 (2001) 064 [hep-th/0105048] [INSPIRE].

[64] A. Pomarol, Gauge bosons in a five-dimensional theory with localized gravity, Phys. Lett. B 486 (2000) 153 [hep-ph/9911294] [INSPIRE].

[65] H. Davoudiasl, J.L. Hewett and T.G. Rizzo, Bulk gauge fields in the Randall-Sundrum model, Phys. Lett. B 473 (2000) 43 [hep-ph/9911262] [INSPIRE].

[66] K. Agashe and A. Delgado, A note on CFT dual of RS model with gauge fields in bulk, Phys. Rev. D 67 (2003) 046003 [hep-th/0209212] [INSPIRE].

[67] M.T. Arun and D. Choudhury, Bulk gauge and matter fields in nested warping: II. Phenomenological consequences, in preparation. 